\title{
Flow-Induced Motions of Flexible Filaments Hanging in Cross-Flow
}

DOI:

10.1016/j.expthermflusci.2018.04.023

\section{Document Version}

Accepted author manuscript

Link to publication record in Manchester Research Explorer

\section{Citation for published version (APA):}

Silva Leon, J., Cioncolini, A., Filippone, A., \& Domingos, M. (2018). Flow-Induced Motions of Flexible Filaments Hanging in Cross-Flow. Experimental Thermal and Fluid Science, 97, 254-269.

https://doi.org/10.1016/j.expthermflusci.2018.04.023

\section{Published in:}

Experimental Thermal and Fluid Science

\section{Citing this paper}

Please note that where the full-text provided on Manchester Research Explorer is the Author Accepted Manuscript or Proof version this may differ from the final Published version. If citing, it is advised that you check and use the publisher's definitive version.

\section{General rights}

Copyright and moral rights for the publications made accessible in the Research Explorer are retained by the authors and/or other copyright owners and it is a condition of accessing publications that users recognise and abide by the legal requirements associated with these rights.

\section{Takedown policy}

If you believe that this document breaches copyright please refer to the University of Manchester's Takedown Procedures [http://man.ac.uk/04Y6Bo] or contact uml.scholarlycommunications@manchester.ac.uk providing relevant details, so we can investigate your claim.

\section{OPEN ACCESS}




\title{
Flow-Induced Motions of Flexible Filaments Hanging in Cross- Flow
}

\author{
Jorge Silva-Leon $^{\mathrm{a}, \mathrm{b}}$, Andrea Cioncolini $^{\mathrm{a}}$, Antonio Filippone $^{\mathrm{a}}$, Marco Domingos $^{\mathrm{C}}$ \\ a School of Mechanical, Aerospace and Civil Engineering, University of Manchester, George Begg Building, Sackville Street, \\ M13 9PL, Manchester, United Kingdom \\ b Escuela Superior Politécnica del Litoral, ESPOL, Facultad de Ingeniería en Mecánica y Ciencias de la Producción, \\ Campus Gustavo Galindo Km 30.5 Vía Perimetral, P.O. Box 09-01-5863, Guayaquil, Ecuador \\ ${ }^{c}$ School of Mechanical, Aerospace and Civil Engineering, University of Manchester, Manchester Institute of Biotechnology, \\ Sackville Street, M13 9PL, Manchester, United Kingdom
}

\begin{abstract}
Experiments were carried out to study the dynamics of hanging cantilever flexible filaments in air cross-flow. Thirteen flexible filaments of $0.61 \mathrm{~mm}$ diameter and lengths from $20 \mathrm{~mm}$ to $60 \mathrm{~mm}$ were tested with wind speeds in the range of $1 \mathrm{~m} / \mathrm{s}$ to $15 \mathrm{~m} / \mathrm{s}$, corresponding to Reynolds numbers of $25<R e_{d}<610$ and reduced velocities in the range of $5<U^{*}<130$. Two synchronized fast-imaging cameras were used to reconstruct the motion of the filaments in three dimensions, and a blend of linear and on-linear timeseries analysis techniques was used to analyze the observed dynamics. Long filaments show a rich dynamics as the wind speed is gradually increased, ranging from small amplitude vibration to large amplitude limitcycle oscillation and to a more complex chaotic motion. However, short filaments only exhibit a small amplitude vibration-like motion throughout the range of wind speeds tested. Turbulent buffeting is identified as the main source of excitation responsible for the observed filaments dynamics. The results highlight the importance of the filament damping ratio, which is modulated by the filament length, as a controlling parameter for the dynamics of flexible filaments in cross flow, in addition to the flow velocity. The Scruton number for these tests correspond to $31<S c<86$.
\end{abstract}

Key words: flexible fluid-structure interaction; filament; turbulence-induced vibration; turbulence buffeting; limit-cycle oscillation

\section{Introduction}

Fluid-structure interaction problems with flexible structures are becoming increasingly important in engineering applications. Examples include flexible marine propellers (Young, 2008), flexible turbomachinery for biomedical applications (Campbell and Paterson, 2011), flapping wing propulsion for micro aerial vehicles (Unger et al., 2012), flexible bladed wind turbines (MacPhee and Beyene, 2016), towing cables (Obligado and Bourgoin, 2013; Wang et al., 2008), 
cable-stayed bridges and antennae (Poulin and Larsen, 2007). Flexible fluidstructure interactions are also ubiquitous in nature: flexible vegetation reconfigures dynamically under the action of wind (de Langre, 2008) or water flow, birds use their feathers as passive flow control devices (Dauptain et al., 2008), and the biology of reproduction in mammals heavily depends on the interaction of flexible structures with a surrounding fluid environment, as the flagellar motion allows the sperm to make its way through the female reproductive tract (Simons et al., 2014; Sniderman, 2016; Wrobel et al., 2016).

In particular, fluid-structure interactions with flexible filaments have received considerable interest in recent years, notably in connection with drag reduction (Alben et al., 2004, 2002; Favier et al., 2009; Niu and Hu, 2011) and energy harvesting applications (Akcabay and Young, 2012; Allen and Smits, 2001; Grouthier et al., 2014; Tang et al., 2009). A pioneering experimental study on flexible filaments in flow was carried out by (Zhang et al., 2000) in a soap film vertical flow. Their test apparatus consisted of a gravity-driven flow of a soap-water mixture that was guided between nylon threads to produce a thin film flow (3-4 microns in thickness) in which they placed their flexible filament aligned with the flow. In particular, they studied the bifurcations in filament behavior as function of the filament length, observing two types of filament motion: stretched-straight (observed with short filaments) and flapping (observed with long filaments). In the stretched-state mode, the filament remained straight and aligned with the flow, while in the flapping mode the filament oscillated harmonically. They found a bistability region at the boundary between these two modes, where the filament switched between the stretched-straight and the flapping states when externally disturbed, i.e. the transition from one type of motion to the other was externally triggered in their experiments. They also studied the interaction between two adjacent filaments in flow, and observed three types of motion when increasing the separation distance between them: in-phase symmetric flapping (the filaments flap synchronized and in phase), out-of-phase symmetric flapping (the filaments flap synchronized but out of phase), and decoupled (each filament flaps at its own pace). Their study motivated the employment of the soap film as a standard experimental technique to study the dynamics of flexible filaments in flow. Ait Abderrahmane et al. (2011) focused on flexible filament dynamics beyond flutter at high flow velocity, observing that the flapping motion was quasiperiodic (more than one dominant frequency), and that the transition to chaotic motion followed a quasiperiodic route. Additionally, they found a switching oscillation between stretched-straight and flapping states that did not require any external triggering perturbation, and was therefore different in nature from the bistability phenomenon observed previously by Zhang et al. (2000) that did require an external trigger. Jia and Yin (2009) employed horizontal and vertical soap films to study the motion of a flexible filament located in the wake of a circular cylinder. They observed that the response modes of the filament were different in the two experimental setups due to the effect of gravity. Schouveiler et al. (2005) experimentally investigated the dynamics of silicone filaments of high mass ratio (i.e. filament density much larger 
than the surrounding fluid density) in a horizontal wind tunnel, focusing on the threshold of critical flutter speed and its theoretical prediction via a linear stability analysis. They argued that the motion of the filament was mainly two-dimensional and attributed the onset of flutter instability to a flow-induced vibration, in contrast with the vortex-induced vibration that normally characterizes the onset of flutter in low mass ratio systems (i.e. structure and surrounding fluid densities comparable) (Facchinetti et al., 2004).

Available numerical studies of flexible filaments in flow (Alben and Shelley, 2008; Favier et al., 2014; Tian et al., 2011; Zhu, 2009; Zhu and Peskin, 2003, 2002) qualitatively confirm the trends observed in experimental studies, and highlight the importance of the filament bending rigidity and filament to fluid density ratio on the dynamics of flexible filaments in flow. In particular, both the decrease of the filament bending rigidity and the increase of filament to fluid density ratio were found to destabilize the filament dynamics, thus promoting a transition from a periodic flapping motion to a chaotic one. In other words, very flexible filaments exposed to a low density fluid flow seem more prone to a chaotic dynamics, whereas periodic flapping characterizes less flexible filaments exposed to a high density fluid flow.

The dynamics of flexible filaments in flow is to a certain extent similar to other slender flexible structures such as pipes and plates exposed to fluid flow. In this regard, Paidoussis (2014) has extensively covered the state-of-the-art knowledge on the linear and nonlinear instabilities arising in slender systems in axial flow, while Paidoussis et al. (2010) have covered the instabilities arising from cross flow. In their study, Modarres-Sadeghi et al. (2008) numerically simulated the onset of three-dimensional motion in horizontal and vertical flexible cantilever pipes with internal fluid flow. They found that both configurations exhibited qualitatively similar dynamics as function of the mass ratio and the dimensionless flow velocity. For low mass ratio, in particular, they found that at the onset of instability the motion of the pipes was three-dimensional and remained like that for increasing dimensionless flow speeds. On the other hand, for high mass ratio they found that the motion was two-dimensional and remained like that with increasing dimensionless flow speeds. For intermediate mass ratio values the motion switched between two- and threedimensional as the dimensionless flow speed was varied. This suggests that the motion of slender flexible structures in fluid flow can be either two- or threedimensional, depending on the mass ratio and flow velocity, and can switch between two- and three-dimensional as the flow speed is gradually changed. According to the authors, the reason why the motion can be either two- or threedimensional is yet unknown, and only confirms the complexity of the dynamics of flexible bodies interacting with fluid flow.

Notwithstanding the investigations carried out so far, the dynamics of flexible filaments in flow is far from being satisfactorily characterized and understood. Most of the available experimental investigations have been carried out in axial flow with 
soap films aligned with gravity, which allow a good experimental resolution at the expense of constraining the filament motion to two-dimensional, and in the only three-dimensional experimental study currently available (Schouveiler et al., 2005) the filament motion was actually resolved in a two-dimensional front-view plane and did not extend beyond the onset of instability. Experimental evidence gathered with other flexible structures exposed to fluid flow suggests that the filament motion could be either two- or three-dimensional, depending on the flow velocity, while available numerical studies show that the filament motion can be either periodic or chaotic, depending on the filament bending rigidity and filament to fluid density ratio. The present study was therefore conducted to broaden the knowledge of the dynamics of flexible filaments in air flow. In particular, we investigated vertical cantilever flexible filaments in horizontal cross flow: a configuration relevant for energy harvesting, for passive flow control, for biological applications and for slender engineering structures such as antennae and marine cables. Threedimensional effects in the filament motion were resolved using two synchronized fast imaging cameras, while the dynamics of the filaments was analyzed using linear and non-linear time series analysis techniques. Besides yielding valuable insight into the dynamics of flexible filaments in flow, the present study provides useful benchmark data for the calibration and fine-tuning of fluid-structure interaction computer codes.

\section{Experiments and Methods}

\subsection{Filaments characterization}

The flexible filaments used in the present study (Fig. 1) were realized using an additive manufacturing system (model: 3D-Bioplotter; make: Envisiontec GMBH) to produce flexible filaments of uniform circular cross-section (to within \pm 10 microns), and smooth surface finishing. The filaments were manufactured from a highly flexible commercial silicone rubber (density $=1.0 \pm 0.05 \mathrm{~g} / \mathrm{cm}^{3}$ ) and were printed following straight paths without bends in order to avoid any curvature or permanent deformations. Notwithstanding the filaments flexibility, no filament elongation and/or permanent deformation were observed during or after the tests. The diameter of the filaments $(D=0.61 \pm 0.02 \mathrm{~mm}$ ) was measured via Scanning Electron Microscopy, while their modulus of elasticity $(E=116 \pm 5 \mathrm{kPa})$, Poisson ratio $(v=0.0101 \pm 0.0004)$ and bending rigidity $\left(B=(1.15 \pm 0.20) \times 10^{-12} \quad \mathrm{Nm}^{2}\right)$ were determined via preliminary tension tests and static deflection tests (described in the Appendix). Additionally, the natural vibration frequencies of the filaments were determined from forced vibration tests, while the damping ratio $(\zeta)$ was determined from free vibration tests in still air (described in the Appendix). The damping ratio is a dimensionless parameter that measures how rapidly free oscillations of the filaments decay after having applied a disturbance. As described in the Appendix, the filament was manually displaced from its vertical equilibrium configuration, liberated from rest and its free damped vibration recorded. The damping ratio was 
then computed following the well-known logarithmic decrement of the envelope. Further details on the filaments fabrication and on their static and dynamic characterization are provided in the Appendix, while Table 1 summarizes the geometrical and dynamical properties measured for the thirteen flexible filaments used in the present study. As can be seen in Table 1, all filaments used in the present study have the same diameter but different lengths varying from $20 \mathrm{~mm}$ to $60 \mathrm{~mm}$ (measured to within $\pm 0.5 \mathrm{~mm}$ ) and corresponding to length to diameter ratios L/D in the range of 98 (Filament 1) down to 33 (Filament 13). For Filaments 1 through 6 it was possible to measure the natural vibration frequencies for the first four modes $\left(f_{n 1}\right.$ to $f_{n 4}$ in Table 1), while the filament displacement was too small to identify higher modes. With Filaments 7 through 11 it was possible to measure the first three modes $\left(f_{n 1}\right.$ to $f_{n 3}$ in Table 1$)$, while only the first two modes were resolvable with the shortest Filaments 12 and $13\left(f_{n 1}\right.$ and $f_{n 2}$ in Table 1$)$. In order to better highlight their dependence on filament length, the measured natural frequencies and damping ratios are displayed in Fig. 2 as function of the filament length. As can be seen, natural vibration frequencies and damping ratios decrease with increasing filament length. In particular, the first mode frequency and the damping ratio saturate and level off for filament lengths above about $50 \mathrm{~mm}$. Notably, the damping ratio of the shortest filament (Filament 13) is almost three times larger than that of the longest filament (Filament 1): as better discussed later on, this marked variation of the damping ratio with filament length is believed to be a key factor in controlling the filament dynamics.

In order to position the filament inside the wind tunnel a support was devised aiming to make it as little intrusive as possible. As schematically shown in Fig. 3, the test piece was realized by introducing the flexible filament extremity into a straight stainless steel support tube (external diameter: $1.05 \mathrm{~mm}$, internal diameter: $0.9 \mathrm{~mm}$, length: $100 \mathrm{~mm}$ ) and fixing with glue cement the filament end to the free end of the support tube so as to reproduce a cantilever boundary condition. The homogeneity of the connection of the filament to the support tube was checked statically by rotating the support horizontally around its axis and verifying that the configuration assumed by the hanging filament remained the same regardless of the rotation position of the support. The length of the support tube was selected in order to position the filament hanging at approximately mid-height inside the wind tunnel test section, while the diameter and thickness of the support tube were large enough to avoid any vibrations or bending during the experiments. As shown in Fig. 3, the support tube was secured to a support plate and was introduced from the top of the wind tunnel so that the filament would hang vertically like a pendulum in cross-flow. During the tests, the filaments typically did not move in the wake of the support tube and remained for most of the time below the support tube due to gravity effects. Nevertheless, the frequency content in the wake of the support tube was measured at the range of flow speeds explored in the present study $(1<\mathrm{U}<15$ $\mathrm{m} / \mathrm{s}$ ). The vortex shedding frequency from the support tube was above $10^{3} \mathrm{~Hz}$, large enough to neglect any effects on the filaments dynamics, which was characterized by frequencies in the range of $5-30 \mathrm{~Hz}$. Moreover, these frequencies 
disappeared from the support tube spectra when performing the measurements right below and downstream of the free end of the support tube, indicating that no disturbances spread below this level into the flow and into the region where the filament was located. Additionally, any vibrations that could possibly be transmitted from the support plate and/or support tube to the flexible filaments were carefully assessed. First, it was observed that the support did not vibrate when subjected to the range of flow speeds employed in this study. Moreover, the natural frequencies of the support tube were estimated (via standard Euler-Bernoulli beam theory) to be 2-3 orders of magnitude larger than the frequencies found in the filament motion frequency spectra. Therefore, any disturbances on the filament dynamics due to the presence of the support tube were considered to be of second order importance and therefore not taken into account, as their potential effect was considered negligible especially on the large displacement motion of the flexible filaments that is the focus of the present study.

\subsection{Wind tunnel flow characterization}

The experiments were performed in a horizontal wind tunnel (make: Armfield) of octagonal cross section with height and width of $350 \mathrm{~mm}$. Flow speed measurements were performed with a calibrated hot-wire anemometer which was preliminary in-situ validated via a standard experiment of vortex shedding from a circular cylinder in cross-flow (see Appendix for details). The hot-wire anemometer was located $200 \mathrm{~mm}$ downstream and $100 \mathrm{~mm}$ below the filament lowest point in order to assure that the flow speed measurements were not affected by the filament motion. The extension of the boundary layer and the uniformity of the velocity profile within the wind tunnel were measured before testing the flexible filaments: the boundary layer extended less than $10 \mathrm{~mm}$ from the walls of the wind tunnel at all wind speeds tested, while the velocity profile across the wind tunnel (excluding the boundary layer) was uniform to within 1\% (i.e. less than $1 \%$ deviation of the normalized speed with respect to the centerline speed). This assures that the flexible filaments were always exposed to a uniform and fully developed velocity profile during the tests. Measurements of the flow turbulence intensity are shown in Fig. 4 as function of the wind speed. As can be seen, the turbulence intensity reaches a minimum of about $0.35 \%$ at around $3.0 \mathrm{~m} / \mathrm{s}$ and then increases at larger wind speeds, leveling off at an approximately constant value of $0.6 \%$ for wind speeds in the range of $5.5-15.0 \mathrm{~m} / \mathrm{s}$.

The wind tunnel flow was further characterized by measuring the integral length scale (or macroscale) of the turbulent eddies $L_{v x}$, which is an approximate measure of the size of the turbulent eddies in the flow that contain most of the turbulence energy. Following common practice (Naudascher and Rockwell, 1994) we assumed homogeneous turbulence, which is normally considered an acceptable approximation in engineering applications. Two main methods have been developed to estimate the integral length scale of turbulent eddies: in the first

method, which is based on the autocorrelation function of the velocity 
measurements (Naudascher and Rockwell, 1994; O'Neill et al., 2004), the integral length scale of turbulence is estimated through the following equation:

$L_{v x}=U \int_{0}^{\infty} R(\tau) d \tau$

Where $U$ is the mean free stream velocity, meaning that the convection speed of the turbulent eddies is assumed to be the mean flow velocity, $R$ is the autocorrelation function of the velocity time series and $\tau$ is the delay time. Operatively, the autocorrelation function is calculated from the measured velocity time series, and the integral in Eq. (1) is evaluated numerically fixing the upper integration limit to the time the decaying autocorrelation signal first reaches zero. The second and alternative method for calculating the integral length scale of the turbulent flow is based on the energy spectra deduced from the velocity measurements (Roach, 1987). Basically, this method consists of finding the energy levels contained at each frequency of the sampled velocity time series and then determining the energy level of the signal as the frequency goes to zero. The length scale can then be estimated from the following relation (Roach, 1987):

$L_{v x}=\left[\frac{E(f) U}{4 \bar{u}^{2}}\right]_{f \rightarrow 0}$

Where $E$ is the energy spectrum of the velocity signal as function of the frequency $f, U$ is the mean flow velocity, and $\bar{u}$ is the mean fluctuating velocity. Operatively, the energy asymptote is determined from averaging the values of the energy spectrum at low frequencies. The results are presented in Fig. 5, where the integral scale of turbulence $L_{v x}$ estimated with the two methods described above is displayed as function of the wind speed. As can be noticed, the two methods give congruent results and indicate that the most energetic turbulent eddies present in the flow range in size between $5-50 \mathrm{~mm}$.

The temperature and pressure in the laboratory during the experiments were $298 \pm 1 \mathrm{~K}$ and $101 \pm 1 \mathrm{kPa}$, respectively.

\subsection{Experimental procedure}

The motion of the flexible filaments was recorded simultaneously in the horizontal and vertical planes using two synchronized digital cameras (make: Panasonic, model: Lumix DMC-FZ200, recording frequency: 200 frames per second, resolution $480 \times 640$ pixels), positioned on the top and front of the wind tunnel, as schematically shown in Fig. 6 . For the remainder of this study, the motion of the flexible filaments projected onto the planes $X-Y$ and $X-Z$ (as schematically shown in Fig. 6) will be referred to as front view and top view, respectively. The motion recorded from the front view is the usual plane investigated in all previous works 
found in the literature, as this contains the direction of gravity. On the other hand, the motion seen in the top view from above the wind tunnel adds another plane that allows studying the three-dimensional configuration of the filament.

The tests on the flexible filaments began with the filament hanging vertically from the ceiling of the wind tunnel and no flow. The wind tunnel fan motor was then started and the wind velocity set to $1.0 \mathrm{~m} / \mathrm{s}$ (minimum stable velocity achievable in the wind tunnel). Noting that the fluid transit time through the wind tunnel was in the range of $0.3 \mathrm{~s}$ to $3 \mathrm{~s}$ (depending on the wind velocity), the video recording started after 30 seconds of having set a wind speed, so as to avoid any transient effects in the recorder videos, and the recording time at each wind velocity was of approximately 60 seconds. The wind velocity was increased progressively in steps of roughly $0.5 \mathrm{~m} / \mathrm{s}$ until reaching the maximum speed $(15-16 \mathrm{~m} / \mathrm{s})$. Then, the speed was decreased gradually down to the minimum. Notably, no hysteresis effects were observed on the filament motion. The optical resolution of the present setup was estimated to be of $0.20 \mathrm{~mm}$, appropriate to resolve the relatively large displacements of the flexible filaments of interest here. The static reconfiguration of flexible filaments at low wind speed (about $1-5 \mathrm{~m} / \mathrm{s}$ ) is described in Silva-Leon et al., 2018, while the focus here is the dynamic response of the flexible filaments, observed with wind speeds within about $5-15 \mathrm{~m} / \mathrm{s}$. The videos obtained from the two cameras were post-processed using the Image Processing Toolbox of Matlab. In particular, three-dimensional configurations of the filaments were reconstructed and displacement time series extracted at different locations along the filament by measuring along the arc length of the reconstructed filament shapes.

\subsection{Data analysis}

In order to exemplify the typical behaviors found and justify the analysis approach adopted in the remainder of this paper, selected results for the longest filament (Filament 1) are presented in Fig. 7. In particular, Fig 7 includes the envelopes of filament motion in the front view and top view and the trajectories of the free end of the filament as seen in the Y-Z plane (see Fig. 6) that corresponds to the view of an observer located downstream of the filament and facing upstream. The results presented in Fig. 7 refer to two different values of the wind speed: a relatively low wind speed of $6.1 \mathrm{~m} / \mathrm{s}$ and a relatively large wind speed of $15.6 \mathrm{~m} / \mathrm{s}$. Irrespective of the wind speed the amplitude of motion varies widely along the filament, being barely noticeable close to the filament fixed end and progressively increasing as the filament free end is gradually approached. Notably, the filament motion amplitude is not the same in the front view and top view. In particular, for the lower wind speed of $6.1 \mathrm{~m} / \mathrm{s}$ the filament motion is mostly two-dimensional and confined to the front view, as the displacement in the top view is barely noticeable, while at the higher wind speed of $15.6 \mathrm{~m} / \mathrm{s}$ the filament motion has become fully threedimensional, as the displacements in the front view and top view are now comparable in size. This qualitatively agrees with the experimental findings of Schouveiler et al. (2005) with cantilever filaments in cross-flow and also with the 
theoretical results obtained by others for flexible structures of high density compared to the surrounding fluid (Bajaj and Sethna, 1984; Modarres-Sadeghi et al., 2008). Considering that the motion of the filament is not always restricted to a plane, the overall measure used here for characterizing the motion at any location along the filament is the total displacement $A_{y z}$ defined as follows:

$A_{y z}=\sqrt{y^{2}+z^{2}}$

Where $y$ and $z$ are the filament displacements observed in the $Y$ and $Z$ directions (see Fig. 6). As such, the total displacement $A_{y z}$ combines into a single number the displacements observed in two orthogonal directions and can therefore be used to characterize both planar and three-dimensional motion. Even though the total displacement can in principle be computed for any position along the filament, we used the filament free-end as representative to analyze the filament dynamics. Being characterized by the largest displacements (and thus by the lowest measuring errors), in the literature the filament free end is most frequently selected for the analysis, and this motivates the approach adopted here. We doublechecked the dynamics at different locations along the filaments and confirmed that within the limits of the present study the dynamics of the filament free end is indeed representative of the dynamics of the entire filament. As a further confirmation, the normalized frequency power spectra generated for Filament 1 for the two wind speed velocities in Fig. $7(6.1 \mathrm{~m} / \mathrm{s}$ and $15.6 \mathrm{~m} / \mathrm{s})$ are shown in Fig. 8. In particular, the dimensionless coordinate $\mathrm{s} / \mathrm{L}$ in the vertical axis of Fig. 8 represents the filament arc length $s$ measured from the filament fixed end divided by the filament total length, so that the limiting values of 0 and 1 correspond to the filament fixed end and free end, respectively. As can be seen, the frequency of motion is always the same along the filament, thus confirming that the dynamics at different locations along the filament is qualitatively the same.

The total displacement $A_{y z}$ time series were studied using standard time series analysis tools (power spectra, autocorrelation plots, root-mean-squared values) as well as using state-space techniques specifically designed for non-linear dynamical systems: an approach recently adopted by Ait Abderrahmane et al. $(2011,2012)$ in the study of filaments and flags. As pointed out by Moon (2004) and Paidoussis (2014), experimental data is invariably subject to disturbances and noise and thus standard time series analysis techniques might give a partial picture of the dynamics of the system being studied, while the combined use of both standard and state-space techniques can provide a more complete picture. In particular, we used the publicly available software package TISEAN (Hegger et al., 1999) to obtain appropriate values of the embedding delay and dimension for the statespace reconstruction via the delayed vectors method (Bradley and Kantz, 2015). As will be shown, the topology of the reconstructed attractor in state-space reveals useful qualitative characteristics of the flexible filaments dynamics that complete and corroborate the information obtained with standard time-series analysis 
techniques. The time series were filtered using the locally projective non-linear noise reduction method included in the TISEAN package, which is specifically designed for non-linear time-series analysis and specifically takes into account the underlying dynamics of the time series (Hegger et al., 1999). As a matter of fact, the noise in the collected signals was rather small so that the effect of this filter on the time-series was most of the times minimal. Other more classic filters for time series analysis, such as moving average filters, were also checked but the nonlinear filter of the TISEAN package was found superior for the present application (lower noise-to-signal rms ratio and smoother reconstructed attractor in statespace).

\section{Results and Discussion}

The complete raw databank is provided in the Electronic Annex (available in the online version of this paper) and includes, for all filaments tested and all wind velocities investigated, the envelopes of motion in the front view and top view and the trajectories of the filament free-end as seen in the $\mathrm{Y}-\mathrm{Z}$ plane (corresponding to the view of an observer located downstream of the filament and facing upstream, see Fig. 6). The Electronic Annex also includes, for all filaments tested and all velocities investigated, the frequency spectra, histograms, the autocorrelation function and the reconstructed attractor in phase-space computed for the total displacement of the filaments free-end. In the following, selected results are presented in condensed format and discussed to analyze the filaments dynamics.

\subsection{Filaments response}

The response diagram for all filaments tested is presented in Fig. 9 (top), where the dimensionless total displacement $A^{*}$ is plotted versus the dimensionless velocity $\mathrm{U}^{*}$. In order to better visualize the trends, the response diagram for a selection of filaments is also presented in Fig. 9 (bottom). In particular, the dimensionless total displacement is defined as $A^{*}=\sqrt{2} A_{r m s} / L$, where $A_{r m s}$ is the root-mean-squared total displacement of the filament free end $A_{y z}$ (Eq. (3)) and $L$ is the filament length. This parameter represents the ratio between the amplitude of the excursions of the free end of the filament relative to its length. As previously noted, the filaments exhibit dynamical properties (damping ratio and natural frequency), which are highly dependent on the filament length: this dimension was therefore selected here as the representative length scale in place of the more conventional diameter typically used with slender bodies in cross-flow. Following common practice, the reduced velocity is defined as $U^{*}=U /\left(f_{n 1} L\right)$, where $U$ is the free stream wind velocity and $f_{n 1}$ is the first mode natural frequency of the filament (from Table 1). The reduced velocity is the ratio between the two speed scales found in this problem: wind speed and filament natural vibration speed. As can be noticed in Fig 8, the filament length clearly affects the filament response. In particular, the response of long filaments (filaments 1 through 6 with lengths 
varying from $35 \mathrm{~mm}$ to $60 \mathrm{~mm}$ ) at low reduced velocity is a small amplitude motion that slowly and gradually increases in amplitude with increasing reduced velocity. As the reduced velocity reaches a threshold the motion amplitude increases sharply, reaching a maximum that can be up to one order of magnitude larger than the amplitude of motion below the threshold. Notably, the reduced velocity threshold is different for each filament and gradually increases with decreasing filament length, while the maximum in motion amplitude is progressively less prominent as the filament length gradually decreases. The maximum in motion amplitude is rather localized with filaments 1 through 5, while with filament 6 it tends to broaden into a rather flat plateau. Beyond the maximum, the motion amplitude decreases gradually as the dimensionless velocity is further increased until it reaches a minimum beyond which the motion amplitude tends to increase again for very large reduced velocities. On the other hand, the response of short filaments (filaments 10 through 13 with lengths varying from $20 \mathrm{~mm}$ to $26.5 \mathrm{~mm}$ ) remains a comparatively smaller amplitude motion at all reduced velocities tested, with amplitude of motion that increases with increasing reduced velocity, though not monotonically, without localized jumps or sharp maxima. Finally, the response of intermediate length filaments (filaments 7 through 9 with lengths varying from $27.5 \mathrm{~mm}$ to $32 \mathrm{~mm}$ ) is to some extent intermediate between those of long and short filaments: small amplitude motion at low reduced velocity, then a change in trend characterized by a localized but not sharp increase in motion amplitude followed by a rather broad plateau at higher reduced velocities. It is evident from Fig. 9 that the change in filament response as the filament length varies is gradual, so that the previous classification into long filaments, short filaments and intermediate length filaments is to some extent arbitrary, and only proposed for the sake of better clarifying the observations.

In conclusion, the filaments response is clearly modulated by the filament length: short filaments have low amplitude response at all reduced velocities, while long filaments have a richer dynamics characterized by both small and large amplitude response, depending on the value of the reduced velocity.

\subsection{Filaments dynamics analysis}

In what follows we concentrate on filaments 1 and 12, which are representative of long filaments (filaments 1 through 6) and short filaments (filaments 10 through 13 ), and analyze in detail their dynamics. The results are indicative of intermediate length filaments too (filaments 7 through 9), which as previously noted have a response intermediate between those of long and short filaments. In particular, the analysis is carried out using the reconstructed attractor in phase-space, the autocorrelation function and the power spectrum, all computed from the time series of the filament free-end total displacement.

The reconstructed attractor in phase-space and the autocorrelation function for filament 1 and for all values of the reduced velocity are presented in Figs. 10 and 
11, while power spectra for selected reduced velocities are presented in Fig. 12. As can be seen, at low reduced velocities $\left(U^{*} \leq 25\right)$ the reconstructed attractor appears like a small blob, the autocorrelation function decay rather fast, and the power spectrum representative of this reduced velocity range in Fig. 12 (i) presents one single and relatively broad peak. These characteristics show that filament 1 motion at low wind speed $\left(U^{*} \leq 25\right)$ is a small-amplitude vibration: a random and not selfsustained motion that decays and does not leave the vicinity of the stable equilibrium corresponding to the static reconfigured filament. For reduced velocities in the range of $28.3 \leq U^{*} \leq 56$ the reconstructed attractor is ring-like and the autocorrelation function is periodic and slowly decaying, indicating that the filament has entered a limit-cycle oscillation. The power spectrum representative of this reduced velocity range in Fig. 12 (ii) shows a proliferation of clear harmonics of the main frequency, which is typical of resonance phenomena. The histograms shown in the Electronic Annex agree with this observation: limit-cycle motions are characterized by bridge-shape distributions, while low-amplitude vibrations are represented as bell-shape histograms. Finally, for high reduced velocities $\left(U^{*} \geq\right.$ 62.7) the ring-like attractor gets thicker and eventually changes its topology evolving into a blob, the autocorrelation function decays rather fast, and the power spectrum representative of this reduced velocity range in Fig. 12 (iii) shows two dominant frequencies: a first peak at $f_{1}=12.3 \mathrm{~Hz}$ and a second peak at $\mathrm{f}_{2}=22.0 \mathrm{~Hz}$, where the two peaks have comparable magnitude and $f_{2}$ is clearly not an integer multiple of $f_{1}$. This shows that as the wind velocity is gradually increased the limitcycle periodic oscillation is progressively corrupted and gradually replaced by a more complex dynamics that becomes gradually chaotic. In particular, the period doubling suggests a quasiperiodic route to chaos characterized by a shift from a period-1 to a period-2 motion that has also been observed in other experiments published in the literature, see e.g. Ait Abderrahmane et al. (2011).

The reconstructed attractor in phase-space and the autocorrelation function for filament 12 and for all values of the reduced velocity are presented in Figs. 13 and 14, while power spectra for selected reduced velocities are presented in Fig. 15. As can be seen, the reconstructed attractor never appear ring-shaped, the autocorrelation function always decays fast while the power spectra present one rather broad peak and an additional secondary peak at high reduced velocities. This shows that filament 12 never enters a limit-cycle oscillation (at least within the range of reduced velocities explored here), and its dynamics always remains a small amplitude random vibration.

In conclusion, based on the present observations a critical filament length can be established to be around $L / D=43$. Below this threshold the dynamics of short filaments is a small amplitude random vibration, with amplitude of motion that tends to increase with increasing reduced velocity without any localized jumps or sharp maxima. On the other hand, the dynamics of filaments with $L / D>43$ remains a small amplitude random vibration only at low reduced velocities. As soon as the reduced velocity reaches a threshold, these filaments enter into a large amplitude 
limit-cycle oscillation that persists for a relatively large range of reduced velocities, until the filaments enter into a chaotic motion via a quasiperiodic route to chaos. The threshold value of the reduced velocity at the onset of the limit-cycle oscillation decreases with increasing filament length, while the amplitude of motion during the limit-cycle oscillation increases with increasing filament length, indicating that their length modulates the attitude of flexible filaments to enter a limit-cycle oscillation.

\subsection{Filaments dynamics interpretation}

It remains to identify the cause of the observed dynamics: in particular what is sustaining the filaments vibration and why the filament length is so influential for observing limit-cycle oscillations. Since the flexible filaments tested here are slender circular cylinders in cross-flow, vortex shedding is clearly a possible source of excitation. The reduced velocity values typical of vortex-induced vibration are in the range of 1 to 15 , when the reduced velocity is based on the cylinder diameter (Naudascher and Rockwell, 1994). In the present case, the reduced velocity based on the filament length ranged between 5 and 130 (Fig. 9). Should the filament diameter be used as characteristic dimension in place of the filament length, this would correspond to figures on the order of $10^{2}-10^{3}$ : well outside the range of values typical for vortex-induced vibration. It is well known, however, that a number of factors affect vortex shedding in addition to the Reynolds number, including the cylinder inclination and end-effects that are particularly relevant in the present case. Before ruling out vortex shedding as a possible source of excitation, therefore, we performed hot-wire measurements in selected locations in the wake of the vibrating filaments in order to measure the vortex shedding frequency and thus properly assess the role of vortex shedding as a potential source of excitation. The results from a typical measurement made for Filament 11 are presented in Fig. 16. As shown, we made measurements at three locations: behind the tube support (ii), behind the filament at mid-length (iii) and behind the filament at the free-end (iv). The tube support is a rigid cylinder in cross-flow, so vortex shedding measurements at location (ii) are directly comparable with literature data for cylinders in cross-flow. On the other hand, vortex shedding measurements at location (iii) incorporate the effect of the filament inclination, while both the filament inclination and end-effects influence the measurements taken at location (iv). Power spectra generated from the hot-wire measurements at these three locations are presented in Fig. 16. As can be seen, the power spectrum at location (ii) has a single peak that nicely matches the vortex shedding frequency $f_{v s}=818 \mathrm{~Hz}$ predicted for the support tube at these conditions via the usual Strouhal-Reynolds number relationship. In the present study the Reynolds number (based on filament diameter), which represents the ratio between inertial and viscous forces, was in the range $300<R e_{d}<610$ for wind speeds large enough for the filaments to move. The vortex shedding frequency $\mathrm{f}_{\mathrm{vs}}=1252 \mathrm{~Hz}$ predicted for the filament taking into account its inclination with respect to the flow (Zdravkovich, 2003) is clearly present in the power spectra generated at locations (iii) and (iv), together with a peak at $f_{0}=7.5 \mathrm{~Hz}$ that corresponds to the dominant frequency in the filament 
vibration. This confirms that the flexible filaments tested here are shedding vortices, and the vortex shedding frequencies are about two orders of magnitude larger that the frequencies that characterize the filaments vibration. Vortex shedding, therefore, can be ruled out as possible source of excitation responsible of the observed filaments dynamics.

Another possibility is that the filaments vibration is externally-induced: it is well known that lightly damped bodies can incur into self-excited and large amplitude motions in a turbulent flow (Naudascher and Rockwell, 1994). In order for turbulence-induced excitation to occur, the length scale of the most energetic turbulent eddies present in the flow should be comparable with the typical length scale of the vibrating body. In our case the characterization of the turbulent flow in the wind tunnel (Fig. 5) shows that the most energetic turbulent eddies range in size between $10-30 \mathrm{~mm}$ for wind velocities within $4-15 \mathrm{~m} / \mathrm{s}$ (excluding low velocities where the filaments statically reconfigure). This length scale is indeed comparable with the length of the filaments, so that turbulent buffeting could be responsible for the fluctuating load that ultimately drives the filaments vibration. As previously noted, the damping ratio of the filaments is a strong function of their length (Fig. 2 right) and long filaments have much lower damping than short filaments. This is noteworthy because it provides an explanation of the observed filaments dynamics: long filaments are lightly damped and therefore exhibit large amplitude limit-cycle oscillations, while short filaments do not due to their comparatively larger damping. The gradual variation of the damping with filament length explains the gradual change in filaments dynamics as the filament length is gradually varied. In conclusion, therefore, turbulent buffeting is identified here as the principal source of excitation responsible for the observed filaments dynamics: limit-cycle oscillations are observed with long and lightly damped filaments, while the bigger damping of short filaments prevents them entering into limit-cycle oscillations.

Finally, the stability diagram for the filaments tested is presented in Fig. 17 as reduced velocity versus Scruton number (or mass-damping number) defined as $\mathrm{Sc}=2 \mathrm{~m} \zeta / \rho_{\mathrm{f}} \mathrm{D}^{2}$, where $\mathrm{m}$ is the filament mass per unit length, $\zeta$ is the damping ratio of the filament, $\rho_{\mathrm{f}}$ is the fluid density and $D$ is the filament diameter. The Scruton number relates the damping level of a system relative to its size, thus is useful for describing the susceptibility of structures to vibrate. As can be seen, limit-cycle oscillations occur for well-delimited values of the reduced velocity and Scruton number (Scruton number $31<S c<86$ ), again suggesting that the filament damping is a key factor for the occurrence of limit-cycle oscillations. In particular, the trend between reduced velocity and Scruton number in Fig. 17 is a direct proportionality, showing that larger reduced velocities are required to sustain limit-cycle oscillations as the filament gets shorter and its damping increases. 


\section{Conclusions}

The dynamics of thirteen flexible filaments of different lengths exposed to air cross flow was investigated using two synchronized fast-imaging cameras to reconstruct the filaments motion in three-dimensions, and employing both linear and non-linear time series analysis techniques to investigate the filaments dynamics. Filaments of $\mathrm{L} / \mathrm{D} \leq 43$ are characterized by a small amplitude vibration-like motion at all wind speeds tested, while filaments of L/D $>43$ exhibit a richer dynamics: a smallamplitude vibration-like motion at low wind speed followed by a large amplitude limit-cycle oscillation that persists for a relatively large range of wind velocities, until the filaments enter into a chaotic motion via a quasiperiodic route to chaos. The threshold value of the reduced velocity at the onset of the limit-cycle oscillation and the amplitude of motion during the limit-cycle oscillation are both modulated by the filament length, which in turn determines the filament damping ratio that is the key factor in controlling the filaments dynamics. It is suggested that the main source of excitation responsible for the observed filaments dynamics is turbulence buffeting, and not the typical vortex-induced vibration found for other slender structures in cross-flow. The capability of long filaments to incur into limit-cycle-like motion is attributed to the lower damping ratio of long filaments as compared to the relatively higher damping ratio of short filaments.

\section{Acknowledgements}

Jorge Silva wishes to thank the National Government of the Republic of Ecuador for funding provided by SENESCYT. Dr. Andrew Kennaugh is gratefully acknowledged for his technical help in setting up the test apparatus. 


$\begin{array}{ll}\text { Nomenclature } & \\ U & \text { Wind speed in the along wind direction (x direction) } \\ L & \text { Filament length } \\ D & \text { Filament diameter } \\ U^{*} & \text { Reduced velocity } \\ A^{*} & \text { Dimensionless amplitude of oscillations } \\ A & \text { Amplitude of motion } \\ X, Y, Z \text { or } x, y, Z & \text { X direction - along wind } \\ & \text { Y direction - cross wind and parallel to gravity direction } \\ \zeta & \text { Z direction - cross wind and perpendicular to gravity } \\ V & \text { Damping ratio } \\ R e & \text { Poisson ratio } \\ S c & \text { Reynolds number } \\ B & \text { Scruton number } \\ E & \text { Bending stiffness } \\ \rho_{f} & \text { Young modulus } \\ L_{v x} & \text { Fluid density } \\ \bar{u} & \text { Turbulence macro length scale } \\ \alpha & \text { mean fluctuating velocity } \\ S t & \text { Angle of filament relative to } x \text { direction } \\ f_{n} & \text { Strouhal number } \\ m & \text { Natural frequency } \\ f_{v s} & \text { Mass per unit length } \\ f_{o} & \text { Frequency of vortex shedding } \\ g & \text { Frequency of oscillations } \\ & \text { Acceleration due to gravity } \\ & \\ \end{array}$




\section{Appendix}

\section{Filament fabrication parameters}

Nozzles of $0.84 \mathrm{~mm}$ ID were used to produce filaments of $0.61 \mathrm{~mm}$ in diameter (extrusion pressure $0.6 \mathrm{MPa}$ and $0.6 \mathrm{~mm} / \mathrm{s}$ deposition speed). All other processing parameters, namely extrusion temperature $(300 \mathrm{~K})$, and nozzle offset from deposition platform $(0.5 \mathrm{~mm})$ were kept constant throughout the process.

\section{Determination of the Young modulus $(E)$ and Poisson ratio $(v)$}

The Young modulus and the Poisson ratio of the filaments were determined with a series of simple elongation tests under variable loading. In particular, filaments of length $\mathrm{L}=70 \mathrm{~mm}$ were held hanging tightly from top while the other end was free. $A$ small paper basket was attached to the free end via a cotton string (the unitary strain of the filament due to the weight of this basket was about 1\%). The reference point for measuring the elongation was the point where the basket was attached. This was the initial configuration, i.e. no deformation, after which small weights of known mass were gently added to the basket and the resulting elongation of the filament was recorded after 30 seconds to allow for the filament to fully stretch. The loads were then carefully removed one by one in order to detect any hysteresis effect. The maximum elongation was kept below $30 \%$ in order to avoid any plastic deformation in the filaments tested. The diameter change due to the axial deformation of the filament was measured using two digital cameras located perpendicular to each other and focused on the mid-section of the hanging filament. Images were taken before and after the small weights were added to the basket. The Poisson ratio was computed via the relationship $v=-\left(D_{f}-D_{i}\right) / D_{i}$, where $D_{f}$ and $D_{i}$ are the measured filament diameters after and before adding a new weight. Image processing was carried out using Matlab. The initial diameter of the filaments was determined from images taken with a Scanning Electron Microscope (SEM). For each load, the axial stress on the filament was determined using a diameter computed through volume conservation, and cross checked with the one computed via the Poisson ratio. Results from one representative elongation test are presented in Fig. A1, where the axial stress is plotted versus the filament unitary deformation. As well known, the slope of the linear part of the data on the stress-strain plot represents the Young Modulus, which equals 111.4 $\mathrm{kPa}$ for the elongation test depicted in Fig. A1. Elongation tests were repeated several times and the values obtained from each of the test repetition were averaged, yielding final figures of $E=116 \pm 5 \mathrm{kPa}$ for the Young modulus (the reference value provided by the silicon manufacturer was $0.3 \mathrm{MPa}$ ) and $v=0.010 \pm 0.001$ for the Poisson ratio. 
Determination of the Bending rigidity $(B)$

The bending rigidity $B$ of the filaments was determined from static deflection tests under self-weight. As shown in Fig. A2, the filament was clamped from one end and was let to hang horizontally and bend under its own weight.

The equilibrium configuration of the filament is the result of the balance between the moment due to filament weight and the resistance offered by the bending rigidity of the filament itself. The filament initially had a length of $70 \mathrm{~mm}$, and was gradually shortened by carefully cutting pieces of approximately $10-15 \mathrm{~mm}$. After each cut, the filament reached a different equilibrium configuration, as shown in Fig. A2. This figure depicts five equilibrium configurations which correspond to five different filament lengths. Images of the filaments configuration were taken with a digital camera and were post-processed using Matlab. The slope variation along the filament was determined from the shape of the filament and the bending rigidity $B$ was subsequently determined from the bending-curvature relationship for

beams, i.e. $\frac{M}{B}=\frac{d \alpha}{d s}$, where the local moment $\mathrm{M}$ is due to the total weight load perpendicular to the local portion along the filament length $s$, i.e. $W \cos \alpha$. The final result for the filaments bending rigidity is $B=(1.15 \pm 0.20) \times 10^{-12} \mathrm{Nm}^{2}$.

\section{Determination of the natural frequencies and damping ratios}

Natural frequencies and damping ratios of the filaments were determined with free and forced vibrations tests using the experimental setup schematically depicted in Fig. A3. The vibration test shaker comprises a loudspeaker connected to a signal generator, a rigid stainless steel connection rod (diameter: $1.0 \mathrm{~mm}$, length: 200 $\mathrm{mm}$ ) cemented at one end to the membrane of the loudspeaker, and a plastic support plate connected at the other end of the rod where the flexible filament was secured. The sinusoidal wave excitation produced by the signal generator induced a horizontal vibration of the loudspeaker membrane, which was then transmitted by the connection rod to the filament support plate. A low-friction guide support was installed to support the connection rod and allow only horizontal displacements of the connection rod. The connection rod was long enough to avoid any direct interactions between the vibrating loudspeaker membrane and the flexible filament through the ambient air. A reference white circle was realized on the support plate to serve as a reference for optically tracking the horizontal vibration of the support plate. High-speed cameras were used to simultaneously track the vibration of the support plate and the motion of the flexible filament.

The free vibration tests were carried out in this setup with the signal generator off. The filament was manually displaced from its vertical equilibrium configuration, liberated from rest and its free damped vibration recorded. A representative example is provided in Fig. A4, which shows the evolution in time of the displacement from equilibrium of the filament free end. The free vibration tests 
allowed obtaining the free vibration damping ratio from the logarithmic decrement of the free-decay amplitudes of motion, yielding values within $(0.024-0.066)$ that are inversely proportional to the filament length, as shown in Table 1.

On the other hand, the lowest-modes natural vibration frequencies of the flexible filaments were determined form the forced vibration tests by visually identifying the external excitation frequencies at which resonance peaks occurred. In particular, the signal generator was set to produce a sinusoidal wave excitation (this was later double-checked using a square signal, which produced comparable results), and the frequencies of the signal were increased in steps of $0.1 \mathrm{~Hz}$ from $1 \mathrm{~Hz}$ up to 25 $\mathrm{Hz}$ (the range of interest for the flow-induced vibrations analyzed here). It was possible to identify the natural vibration frequencies of the first four modes of Filaments 1-6, of the first three modes of Filament 7-11, and of the first two modes of Filament 12-13. The natural vibration frequencies are provided in Table 1 and for each mode are inversely proportional to the filament length, which is consistent with classic beam theory. The first four vibration modes for the filament of free length of $L=60 \mathrm{~mm}$ (representative of Filament 1 in Table 1) are qualitatively the same as the corresponding ones for a rigid cantilever beam, as shown in Fig. A5.

\section{Validation of the constant temperature hot-wire anemometer measurements}

Flow speed measurements were performed with a calibrated hot-wire anemometer which was validated in-situ via a preliminary experiment of vortex shedding frequency from a circular cylinder in cross-flow. The straight hot-wire was located $100 \mathrm{~mm}$ downstream of a circular cylinder of diameter $15.5 \mathrm{~mm}$ which was set in cross flow configuration across the wind tunnel test section. The probe was then set $30 \mathrm{~mm}$ offset from the axis of the cylinder to detect vortex shedding. The measurements are presented in Fig. A6, where the cylinder Strouhal number is displayed as function of the cylinder Reynolds number. The measurements compare well with the following well-known correlation (Blevins, 1990):

$$
S t=0.198\left(1-\frac{19.7}{R e}\right)
$$




\section{References}

Ait Abderrahmane, H., Paidoussis, M.P., Fayed, M., Ng, H.D., 2011. Flapping dynamics of a flexible filament. Phys. Rev. E 84, 66604. doi:10.1103/PhysRevE.84.066604

Akcabay, D.T., Young, Y.L., 2012. Hydroelastic response and energy harvesting potential of flexible piezoelectric beams in viscous flow. Phys. Fluids 24 , 54106. doi:10.1063/1.4719704

Alben, S., Shelley, M., Zhang, J., 2004. How flexibility induces streamlining in a two-dimensional flow. Phys. Fluids 16, 1694-1713. doi:10.1063/1.1668671

Alben, S., Shelley, M., Zhang, J., 2002. Drag reduction through self-similar bending of a flexible body. Nature 420,479-481. doi:10.1038/nature01232

Alben, S., Shelley, M.J., 2008. Flapping States of a Flag in an Inviscid Fluid: Bistability and the Transition to Chaos. Phys. Rev. Lett. 100. doi:10.1103/PhysRevLett.100.074301

Allen, J.J., Smits, A.J., 2001. Energy Harvesting Eel. J. Fluids Struct. 15, 629-640. doi:10.1006/jls.2000.0355

Bajaj, A.K., Sethna, P.R., 1984. Flow Induced Bifurcations to Three-Dimensional Oscillatory Motions in Continuous Tubes 44, 270-286.

Blevins, R.D., 1990. Flow-induced vibration. Van Nostrand Reinhold Co., Inc., New York, NY (USA).

Bradley, E., Kantz, H., 2015. Nonlinear time-series analysis revisited. Chaos 25. doi:10.1063/1.4917289

Campbell, R.L., Paterson, E.G., 2011. Fluid-structure interaction analysis of flexible turbomachinery. J. Fluids Struct. 27, 1376-1391.

doi:10.1016/j.jfluidstructs.2011.08.010

Dauptain, A., Favier, J., Bottaro, A., 2008. Hydrodynamics of ciliary propulsion. J. Fluids Struct. 24, 1156-1165. doi:10.1016/j.jfluidstructs.2008.06.007

de Langre, E., 2008. Effects of Wind on Plants. Annu. Rev. Fluid Mech. 40, 141168. doi:10.1146/annurev.fluid.40.111406.102135

Facchinetti, M.L., de Langre, E., Biolley, F., 2004. Vortex-induced travelling waves along a cable. Eur. J. Mech. - B/Fluids 23, 199-208. doi:10.1016/j.euromechflu.2003.04.004

Favier, J., Dauptain, A., Basso, D., Bottaro, A., 2009. Passive separation control using a self-adaptive hairy coating. J. Fluid Mech. 627, 451. 
doi:10.1017/S0022112009006119

Favier, J., Revell, A., Pinelli, A., 2014. A Lattice Boltzmann - Immersed Boundary method to simulate the fluid interaction with moving and slender flexible objects. J. Comput. Phys. 261, 145-161. doi:10.1016/j.jcp.2013.12.052

Grouthier, C., Michelin, S., Bourguet, R., Modarres-Sadeghi, Y., de Langre, E., 2014. On the efficiency of energy harvesting using vortex-induced vibrations of cables. J. Fluids Struct. 49, 427-440. doi:10.1016/j.jfluidstructs.2014.05.004

Hegger, R., Kantz, H., Schreiber, T., 1999. Practical implementation of nonlinear time series methods: The TISEAN package. Chaos 9, 413-435.

doi:10.1063/1.166424

Jia, L.-B., Yin, X.-Z., 2009. Response modes of a flexible filament in the wake of a cylinder in a flowing soap film. Phys. Fluids 21, 101704.

doi:10.1063/1.3257294

MacPhee, D.W., Beyene, A., 2016. Fluid-structure interaction analysis of a morphing vertical axis wind turbine. J. Fluids Struct. 60, 143-159. doi:10.1016/j.jfluidstructs.2015.10.010

Modarres-Sadeghi, Y., Païdoussis, M.P., Semler, C., 2008. Three-dimensional oscillations of a cantilever pipe conveying fluid. Int. J. Non. Linear. Mech. 43, 18-25. doi:10.1016/j.jnonlinmec.2007.09.005

Naudascher, E., Rockwell, D., 1994. Flow-Induced Vibrations: an Engineering Guide. Dover Publications, Inc.

Niu, J., Hu, D.L., 2011. Drag reduction of a hairy disk. Phys. Fluids 23, 101701. doi:10.1063/1.3639133

O'Neill, P.L., Nicolaides, D., Honnery, D.R., Soria, J., 2004. Autocorrelation Functions and the Determination of Integral Length with Reference to Experimental and Numerical Data. 15th Australas. Fluid Mech. Conf. 1, 1-4.

Obligado, M., Bourgoin, M., 2013. An experimental investigation of the equilibrium and stability of long towed cable systems. New J. Phys. 15, 43019. doi:10.1088/1367-2630/15/4/043019

Paidoussis, M.P., 2014. Fluid-Structure Interactions: Slender Structures and Axial Flow, Volume 1, Second. ed. Academic Press.

Paidoussis, M.P., Price, S., de Langre, E., 2010. Fluid-Structure Interactions: Cross-Flow-Induced Instabilities. Cambridge University Press, Cambridge.

Poulin, S., Larsen, A., 2007. Drag loading of circular cylinders inclined in the alongwind direction. J. Wind Eng. Ind. Aerodyn. 95, 1350-1363. 
doi:10.1016/j.jweia.2007.02.011

Roach, P.E., 1987. The generation of nearly isotropic turbulence by means of grids. Int. J. Heat Fluid Flow 8, 82-92. doi:10.1016/0142-727X(87)90001-4

Schouveiler, L., Eloy, C., Le Gal, P., 2005. Flow-induced vibrations of high mass ratio flexible filaments freely hanging in a flow. Phys. Fluids 17, 47104. doi:10.1063/1.1878292

Silva-Leon, J., Cioncolini, A., Filippone, A., 2018. Determination of the normal fluid load on inclined cylinders from optical measurements of the reconfiguration of flexible filaments in flow. J. Fluids Struct. 76, 488-505.

doi:10.1016/j.jfluidstructs.2017.10.009

Simons, J., Olson, S., Cortez, R., Fauci, L., 2014. The dynamics of sperm detachment from epithelium in a coupled fluid-biochemical model of hyperactivated motility. J. Theor. Biol. 354, 81-94. doi:10.1016/j.jtbi.2014.03.024

Sniderman, D., 2016. Biofluids of reproduction: oscillators, viscoelastic networks, and sticky situations. SIAM News 49(7) 1-4.

Tang, L., Païdoussis, M.P., Jiang, J., 2009. Cantilevered flexible plates in axial flow: Energy transfer and the concept of flutter-mill. J. Sound Vib. 326, 263276. doi:10.1016/j.jsv.2009.04.041

Tian, F.-B., Luo, H., Zhu, L., Liao, J.C., Lu, X.-Y., 2011. An efficient immersed boundary-lattice Boltzmann method for the hydrodynamic interaction of elastic filaments. J. Comput. Phys. 230, 7266-7283. doi:10.1016/j.jcp.2011.05.028

Unger, R., Haupt, M.C., Horst, P., Radespiel, R., 2012. Fluid-structure analysis of a flexible flapping airfoil at low Reynolds number flow. J. Fluids Struct. 28, $72-$ 88. doi:10.1016/j.jfluidstructs.2011.08.009

Wang, F., Huang, G.L., Deng, D.H., 2008. Steady state analysis of towed marine cables. J. Shanghai Jiaotong Univ. 13 E, 239-244. doi:10.1007/s12204-0080239-9

Wrobel, J.K., Lynch, S., Barrett, A., Fauci, L., Cortez, R., 2016. Enhanced flagellar swimming through a compliant viscoelastic network in Stokes flow. J. Fluid Mech. 792, 775-797. doi:10.1017/jfm.2016.99

Young, Y.L., 2008. Fluid-structure interaction analysis of flexible composite marine propellers. J. Fluids Struct. 24, 799-818.

doi:10.1016/j.jluidstructs.2007.12.010

Zdravkovich, M.., 2003. Flow around Circular Cylinders, Vol. 2: Applications. Oxford University Press. 
Zhang, J., Childress, S., Libchaber, A., Shelley, M., 2000. Flexible filaments in a flowing soap film as a model for one-dimensional flags in a two-dimensional wind. Nature 408, 835-839. doi:10.1038/35048530

Zhu, L., 2009. Interaction of two tandem deformable bodies in a viscous incompressible flow. J. Fluid Mech. 635, 455-475. doi:10.1017/S0022112009007903

Zhu, L., Peskin, C.S., 2003. Interaction of two flapping filaments in a flowing soap film. Phys. Fluids 15, 1954. doi:10.1063/1.1582476

Zhu, L., Peskin, C.S., 2002. Simulation of a Flapping Flexible Filament in a Flowing Soap Film by the Immersed Boundary Method. J. Comput. Phys. 179, 452468. doi:10.1006/jcph.2002.7066 


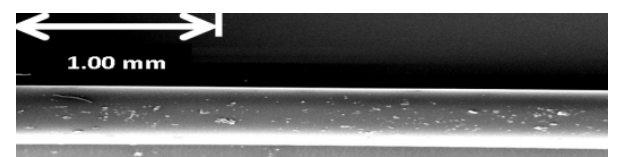

Fig. 1 Flexible filament along with a 5 pence coin and a ruler in $\mathrm{cm}$ (left), and SEM image detail of the filament surface finishing (right). 

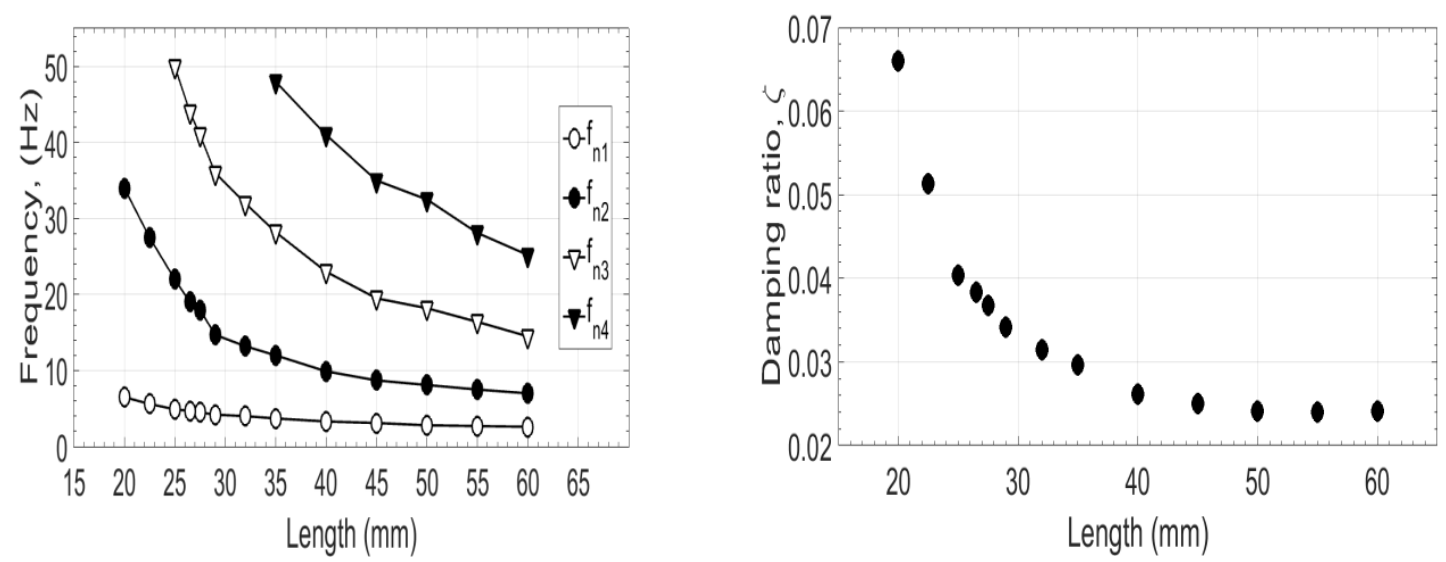

Fig. 2 (left) natural frequencies vs filament length; (right) damping ratio vs filament length. 


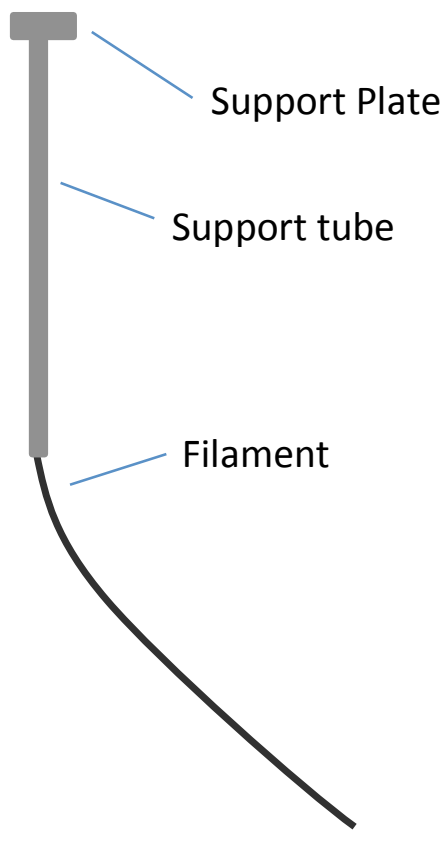

Fig. 3 Schematic representation of the test piece. 


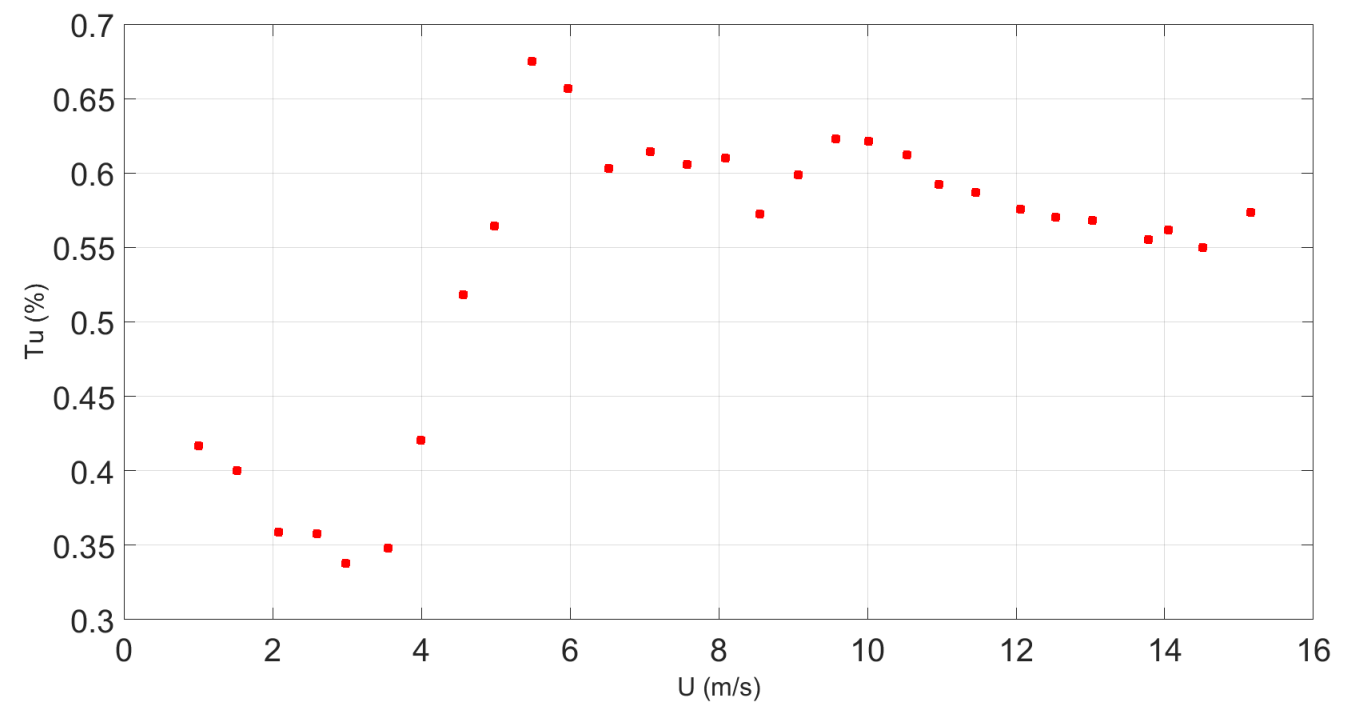

Fig. 4 Turbulence intensity of the free stream flow in the wind tunnel as function of the wind speed. 


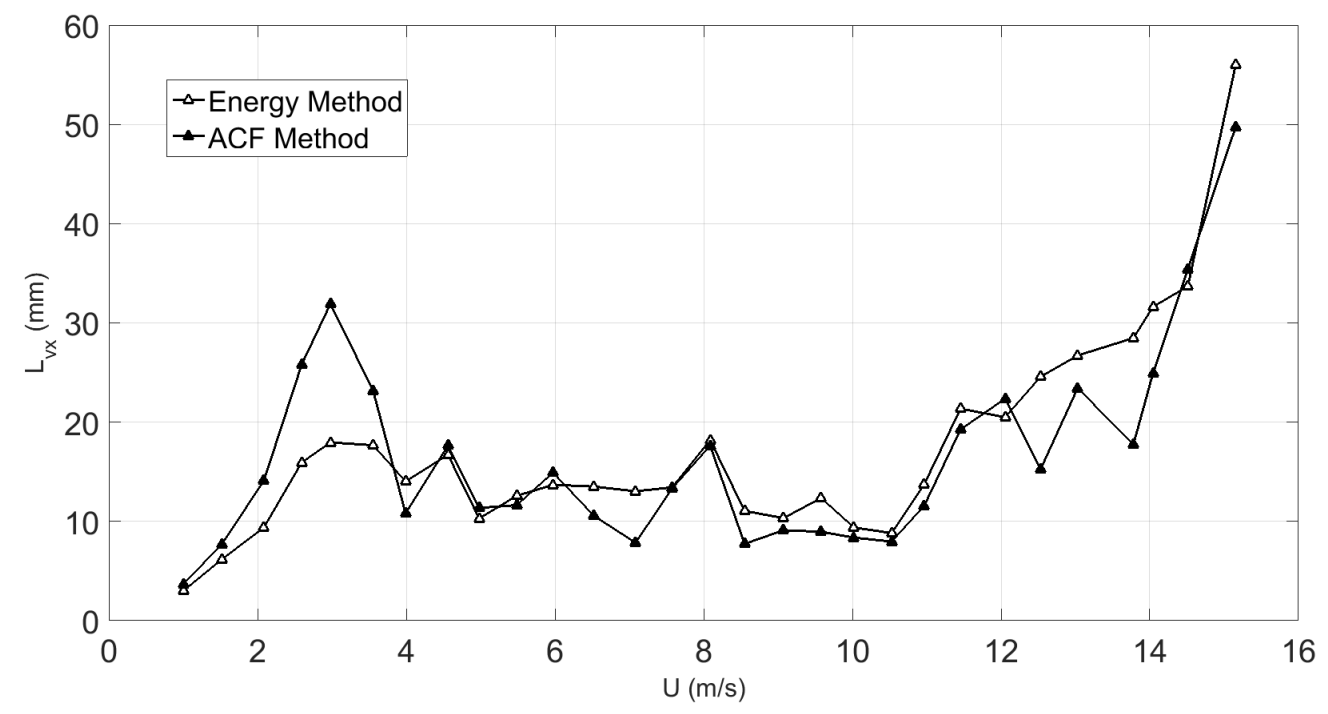

Fig. 5 Integral length scale of the turbulent flow in the wind tunnel for the range of flow speeds tested. 
i)

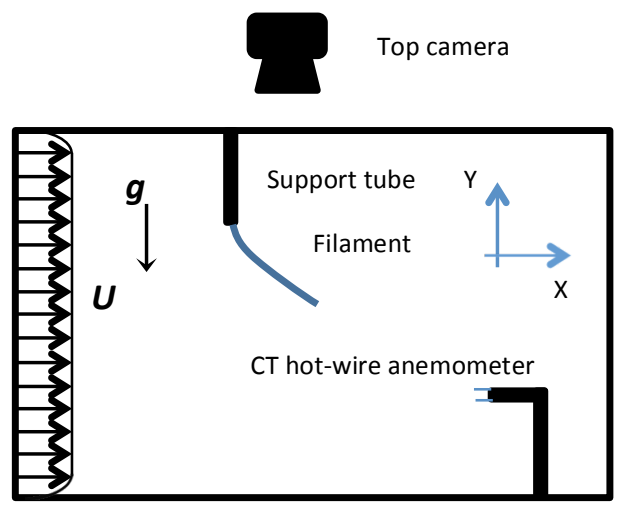

ii)

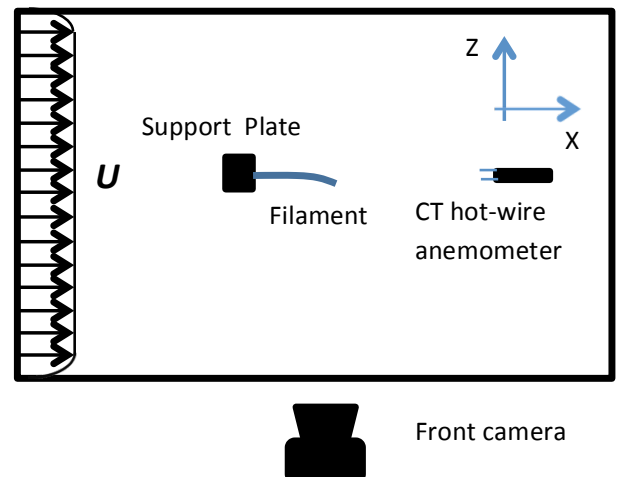

Fig. 6 Schematic representation of the experimental setup: i) front view, plane $X-Y$; ii) top view, plane $\mathrm{X}-\mathrm{Z}$. 
$\mathrm{U}=6.1 \mathrm{~m} / \mathrm{s}$
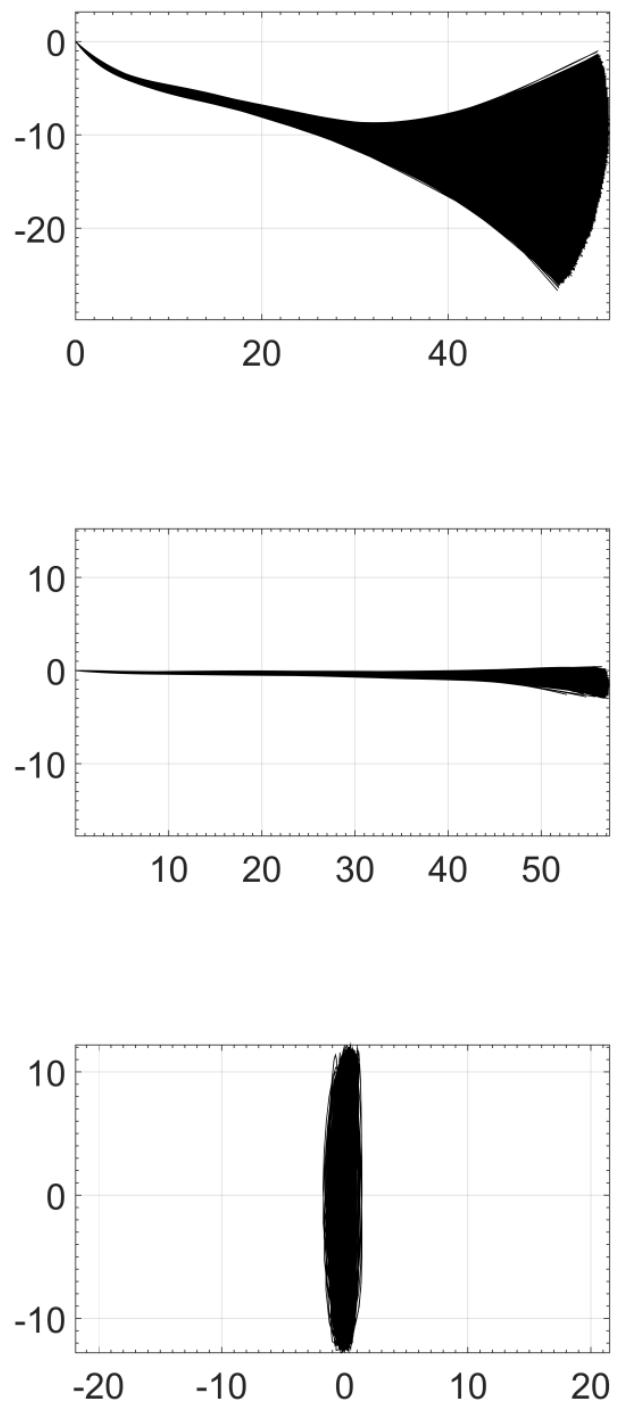

$\mathrm{U}=15.6 \mathrm{~m} / \mathrm{s}$
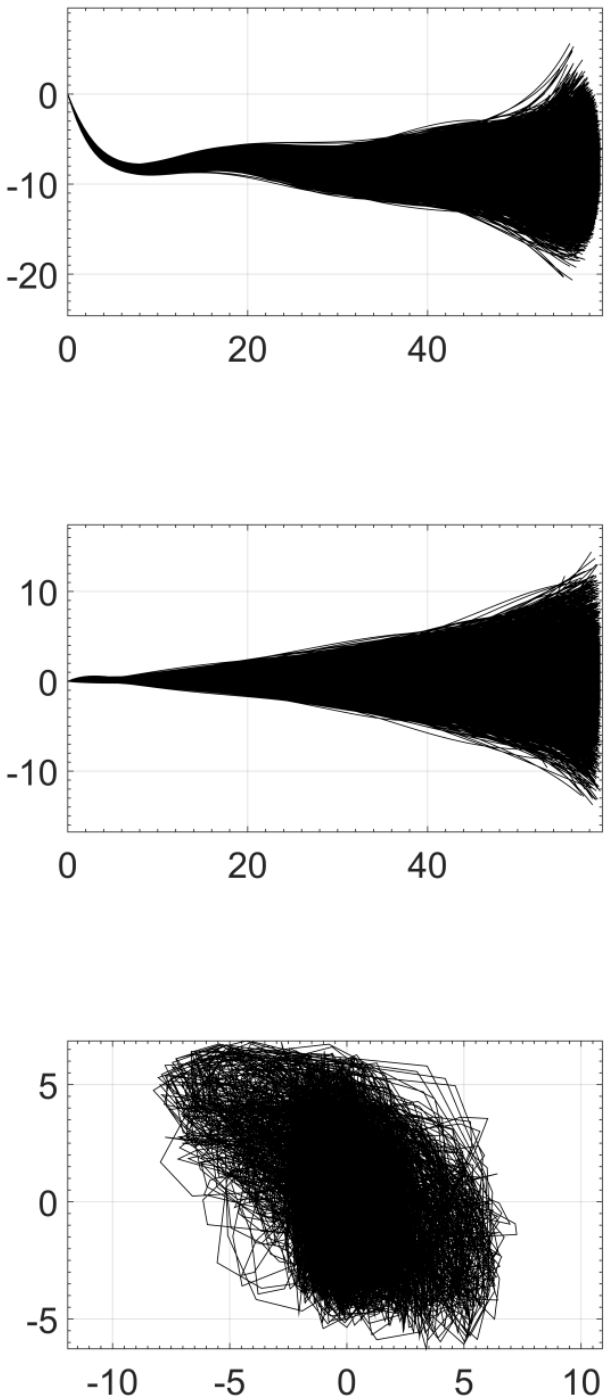

Fig. 7 Raw results for filament 1 at two wind speeds, corresponding to $60 \mathrm{~s}$ of recordings (axes units in $\mathrm{mm}$ ): (upper) envelopes of motion in front view; (middle) envelopes of motion in top view; (bottom) trajectories of filament free end in $Y-Z$ plane. 
i)

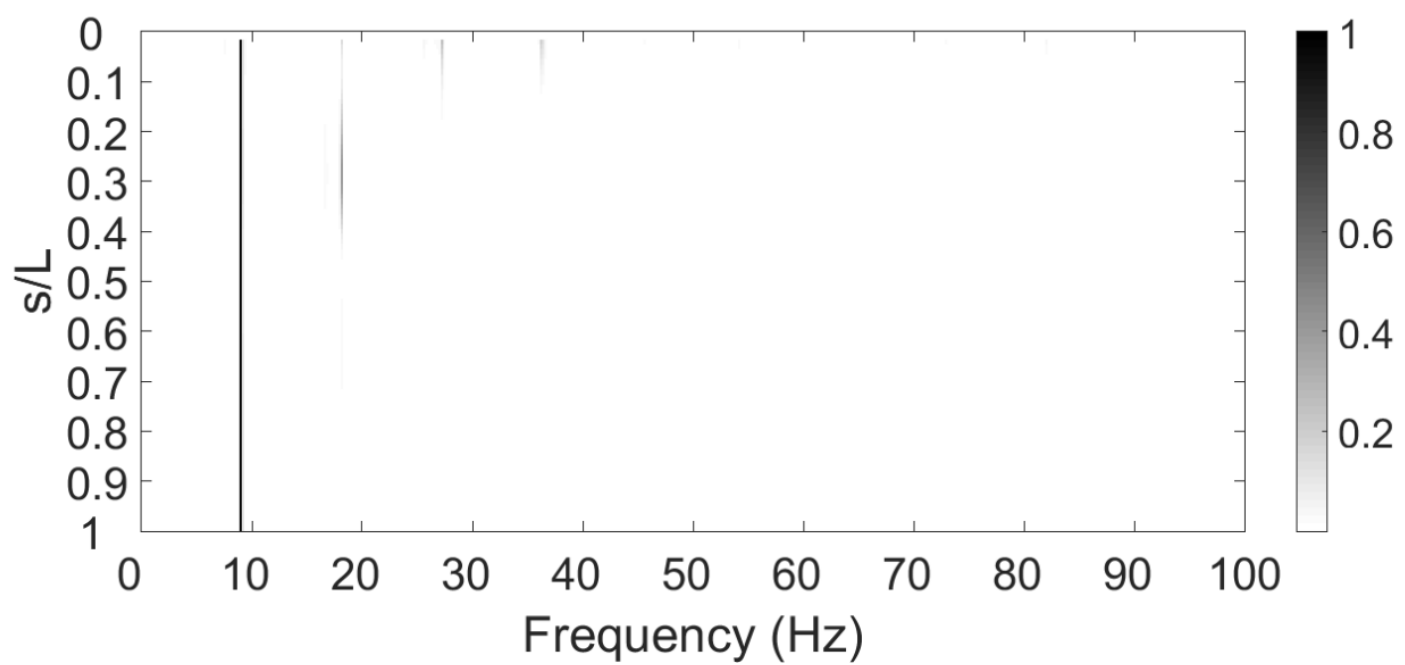

ii)

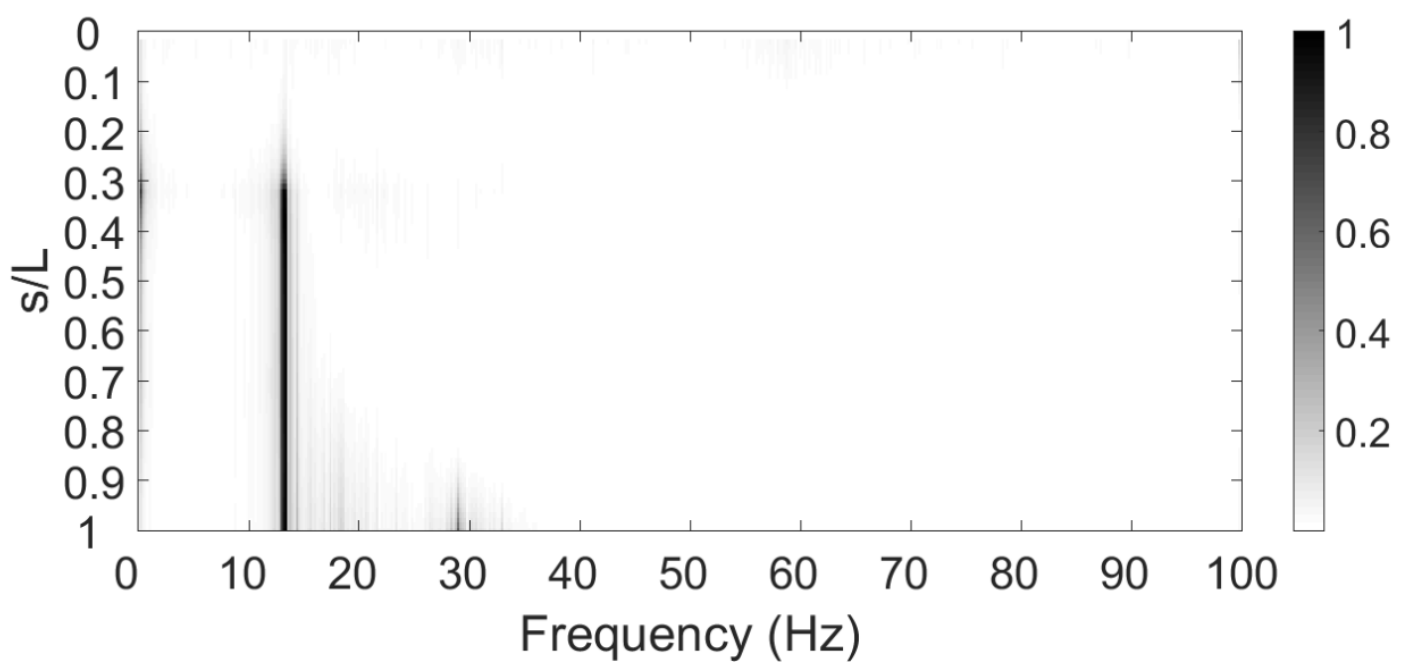

Fig. 8 Normalized PSD of total displacement along Filament 1 at two wind speeds: a) $\mathrm{U}=7.4 \mathrm{~m} / \mathrm{s}$ (frequency of $10.6 \mathrm{~Hz}$ ); b) $\mathrm{U}=15.6 \mathrm{~m} / \mathrm{s}$; (frequency of $13.4 \mathrm{~Hz}$ ) 

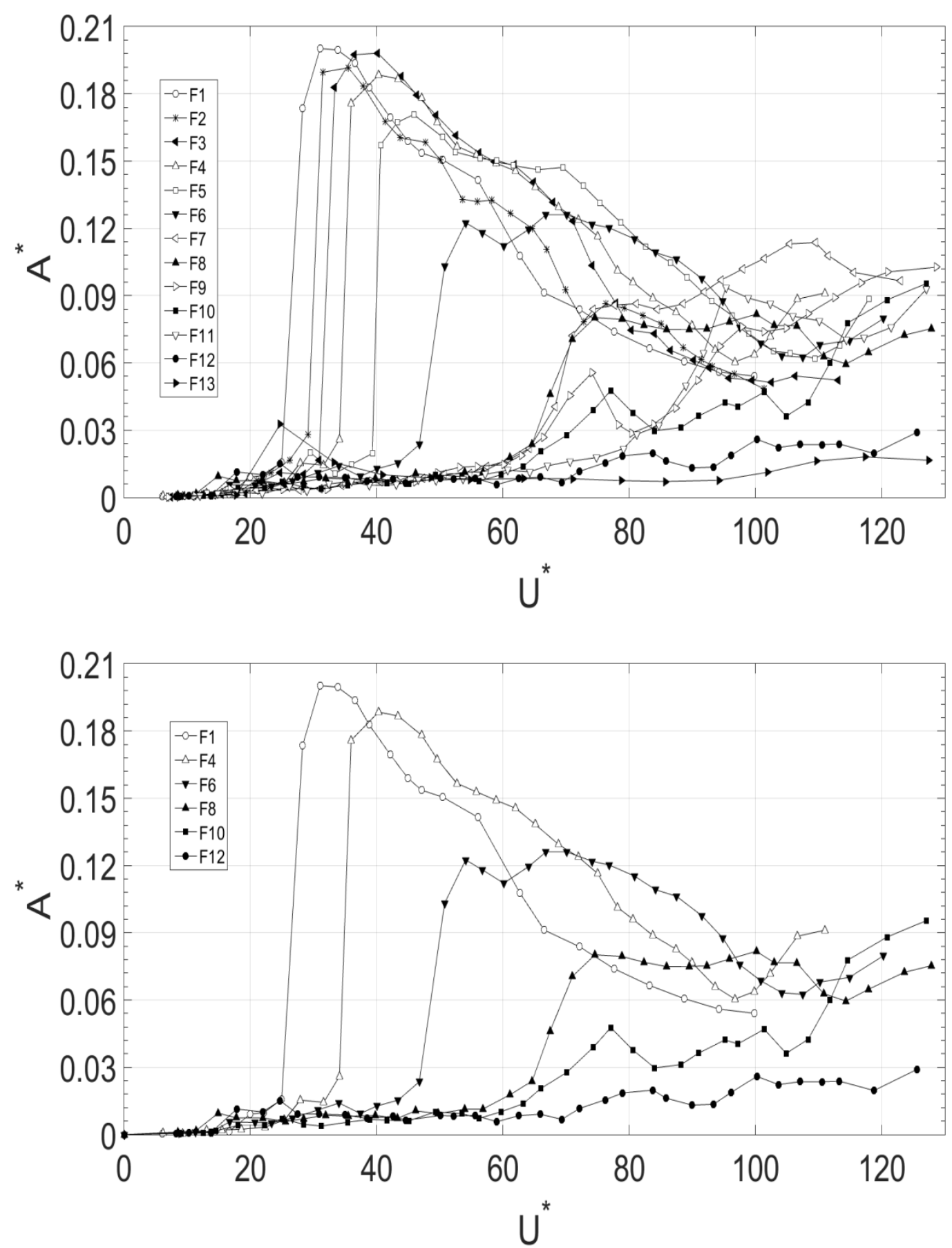

Fig. 9 Dimensionless response diagram of filaments free-end vs reduced velocity (in the legend F1 stands for filament 1, F2 for filament 2, and so forth): all filaments tested (top) and selection of filaments (bottom). 

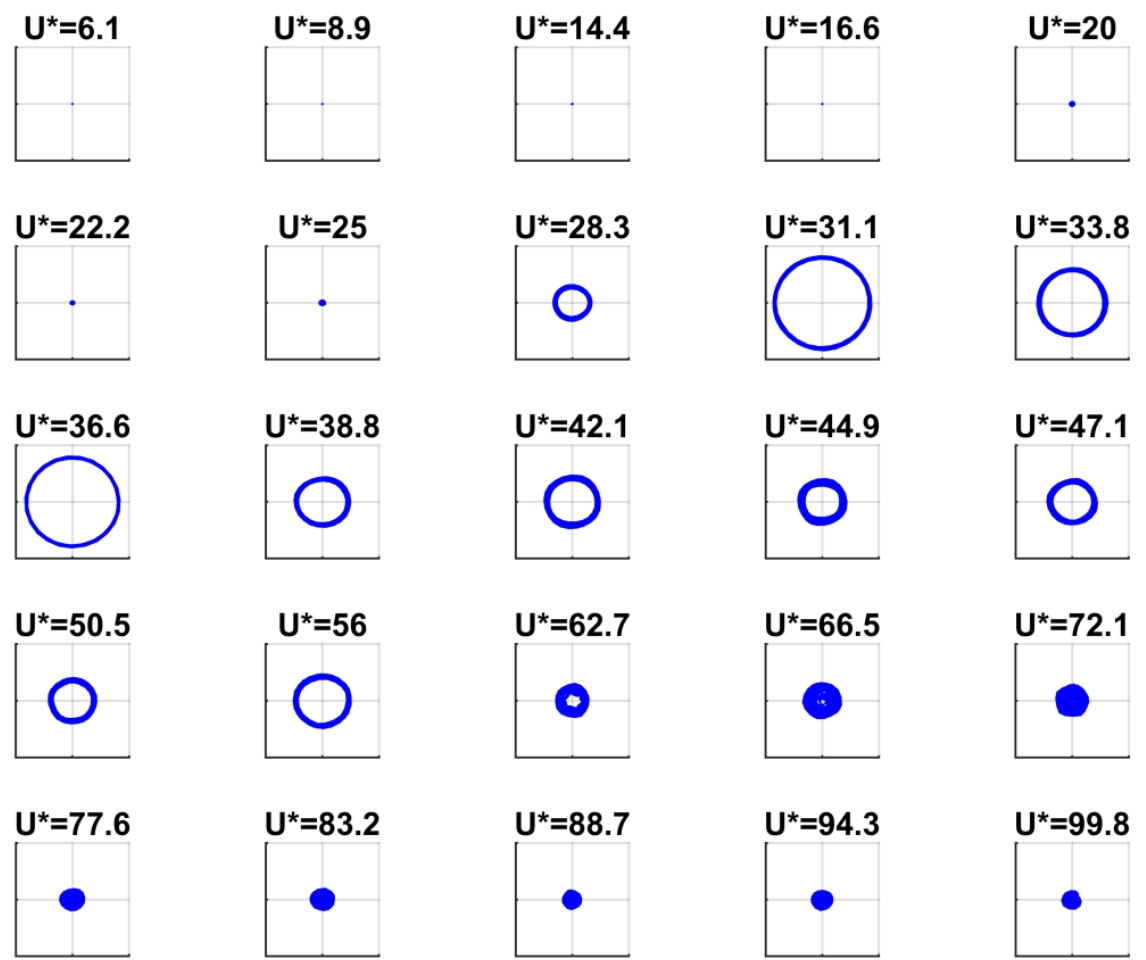

Fig. 10 Reconstructed attractors in phase-space for the free end of Filament 1 in the Y-Z plane. Horizontal Axes: $r_{y z}(t)$, Vertical Axes: $r_{y z}(t+\tau)$. (tau is the optimal delay estimated with the TISEAN software). 

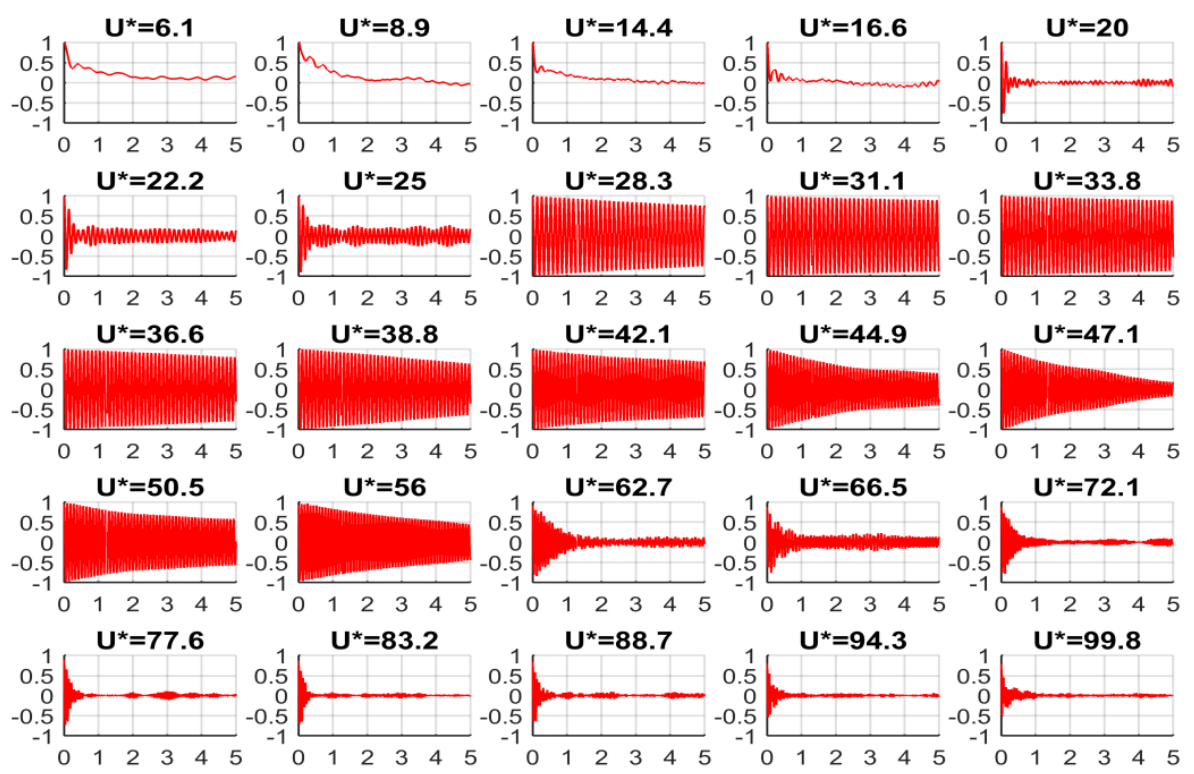

Fig. 11 Autocorrelation function of the displacements of the free end of Filament 1 in the $\mathrm{Y}-\mathrm{Z}$ plane (horizontal axis unit is $\mathrm{s}$ ). 
i) $\quad U^{*}=22.2$

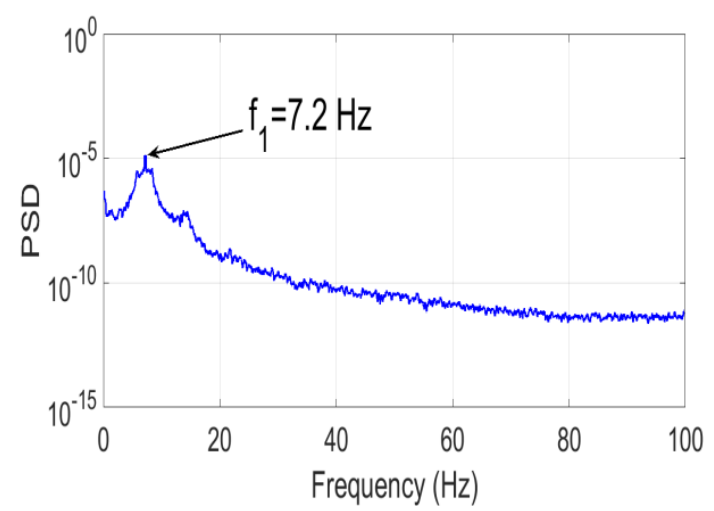

iii) $\quad U^{*}=66.5$ ii) $\quad U^{*}=33.8$

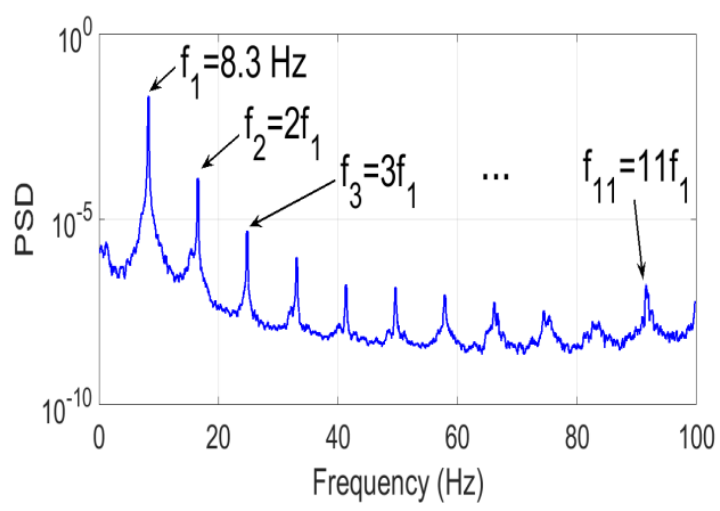

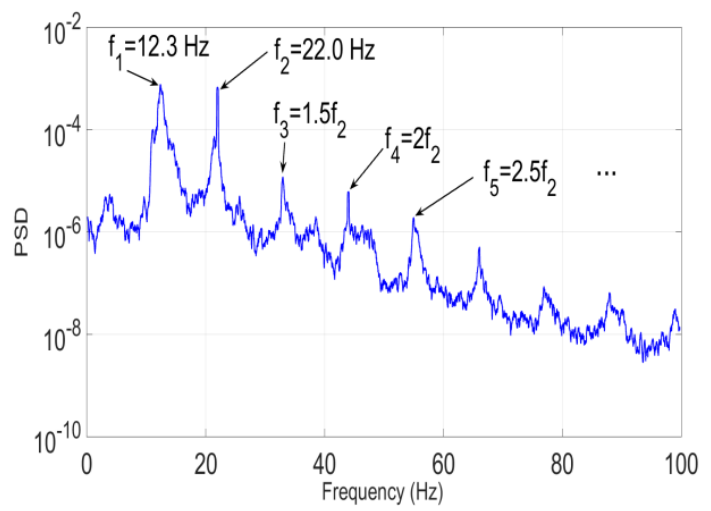

Fig. 12 PSD of displacements of the free end of Filament 1 in the $Y-Z$ plane. i) $U^{*}=22.2$; ii) $U^{*}=33.8$; iii) $U^{*}=66.5$; iv) $U^{*}=99.8$. 


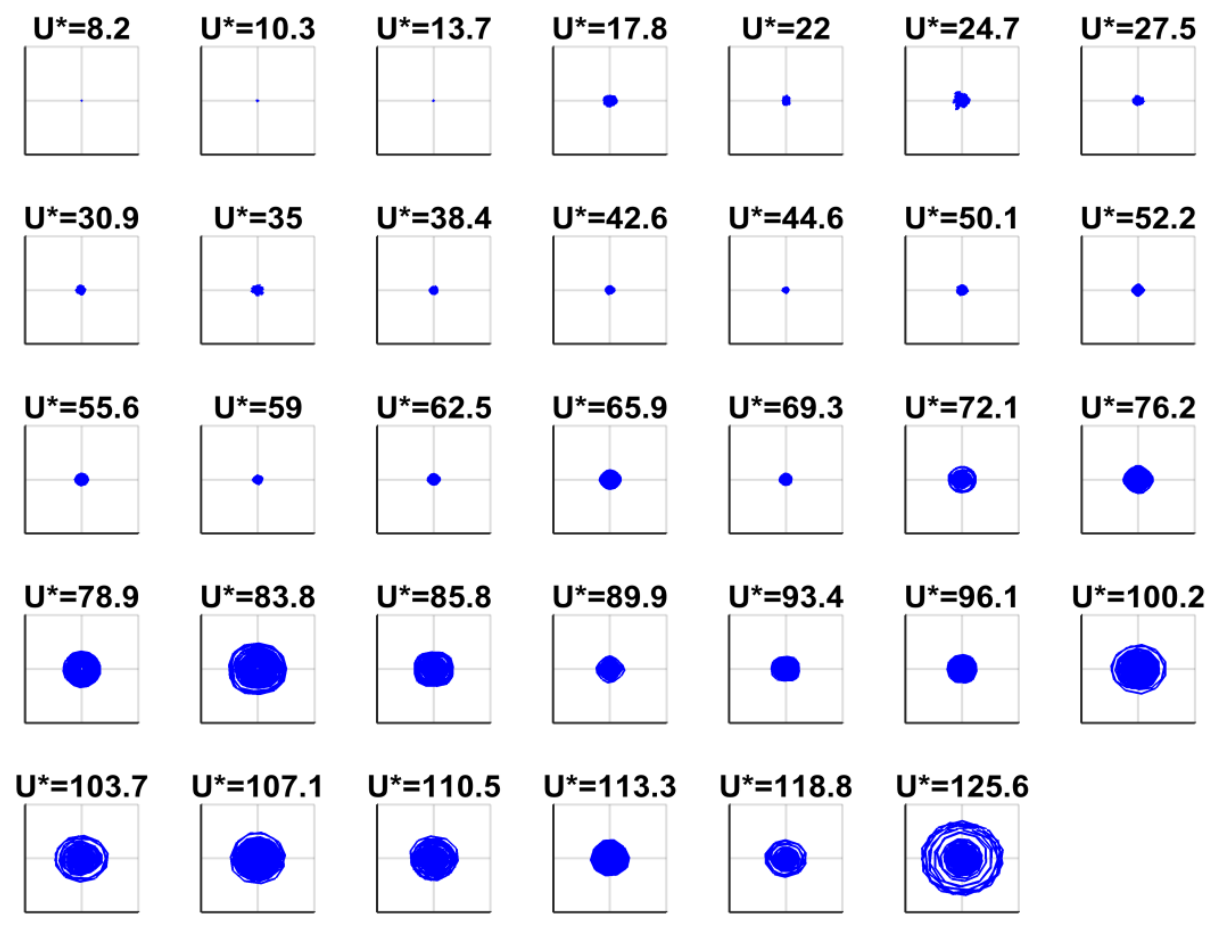

Fig. 13 Reconstructed attractors in phase-space for the free end of Filament 12 in the $Y-Z$ plane. Horizontal Axes: $r_{y z}(t)$, Vertical Axes: $r_{y z}(t+\tau)$. 


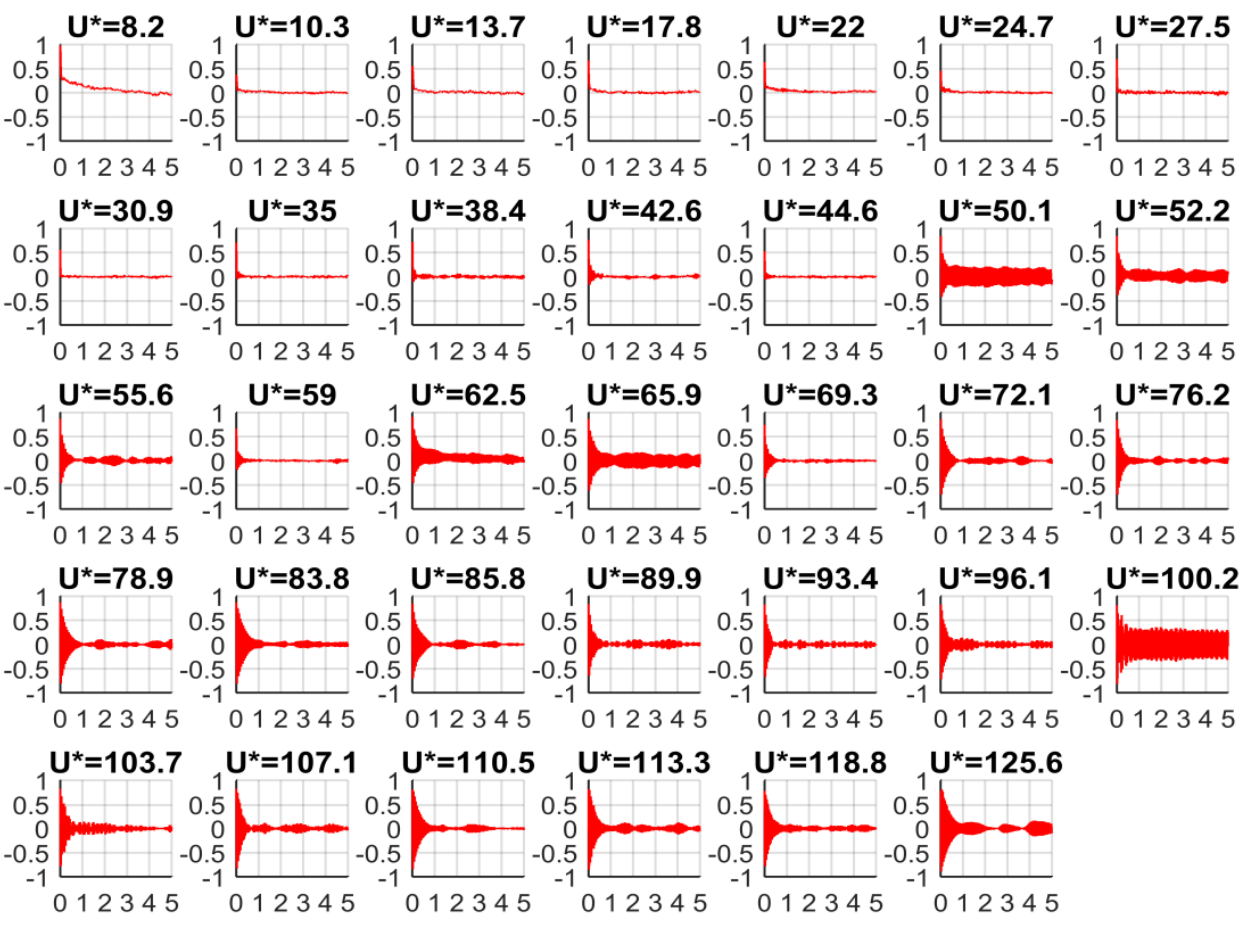

Fig. 14 Autocorrelation function of the displacements of the free end of Filament 12 in the $\mathrm{Y}-\mathrm{Z}$ plane (horizontal axis unit is $\mathrm{s}$ ). 

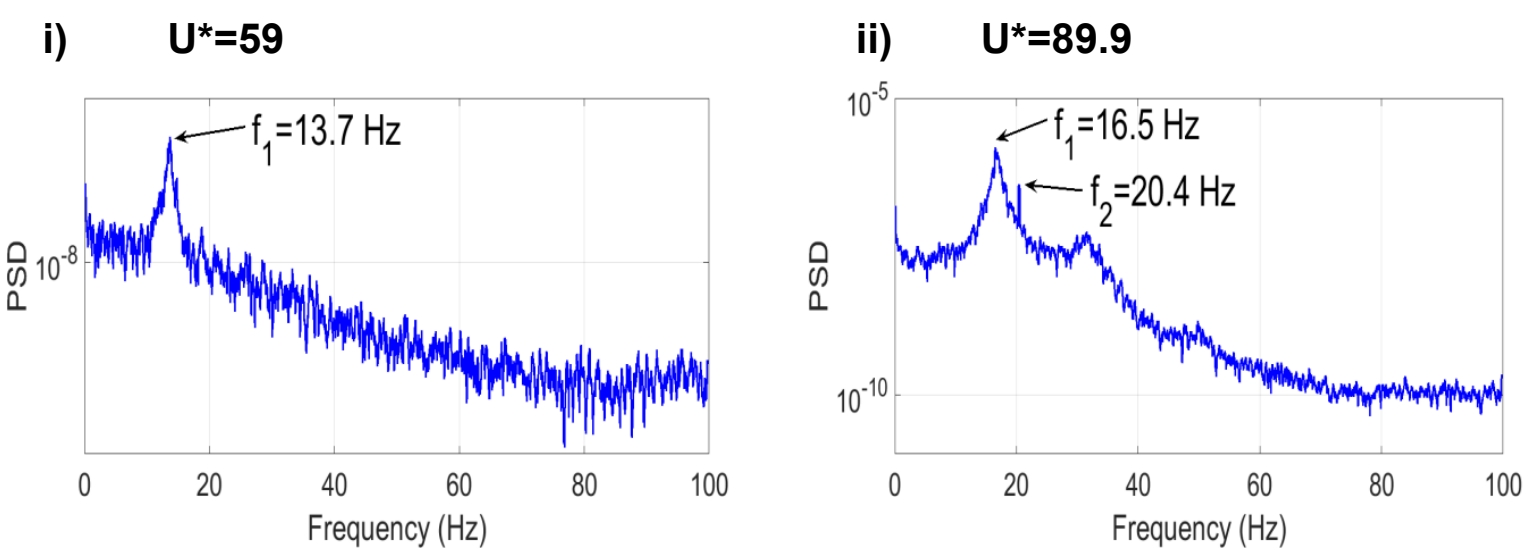

iii) $\quad U^{*}=118.8$

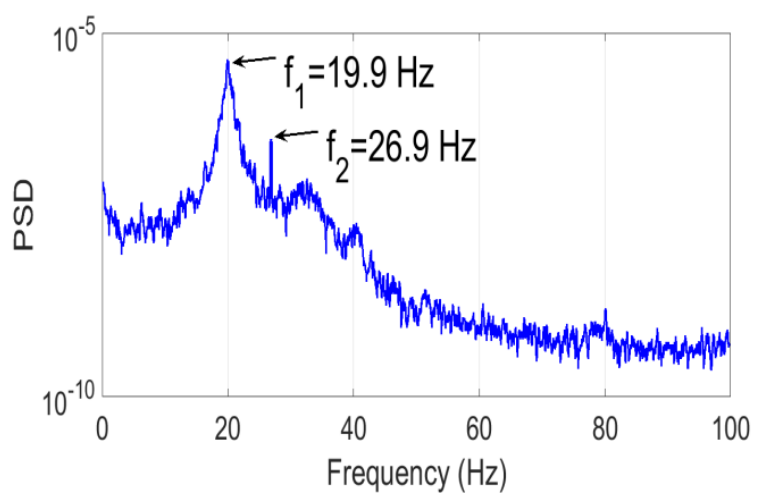

Fig. 15 PSD of displacements of the free end of Filament 12 in the $Y-Z$ plane. i) $U^{*}=59$; ii) $U^{*}=89.9$; iii) $U^{*}=118.8$. 
i)

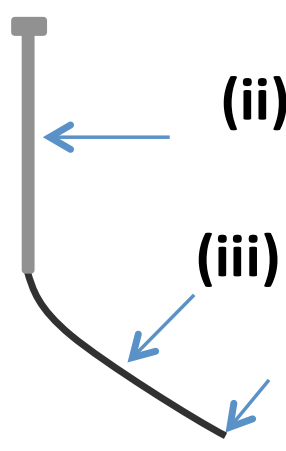

iii)

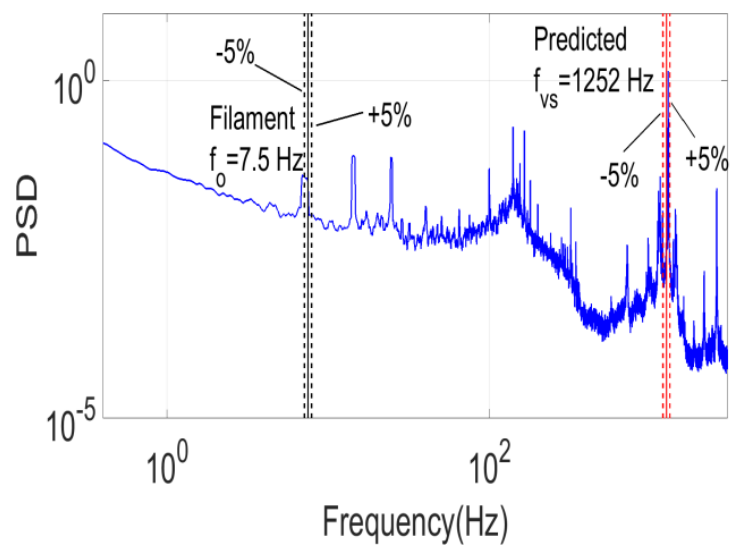

ii)

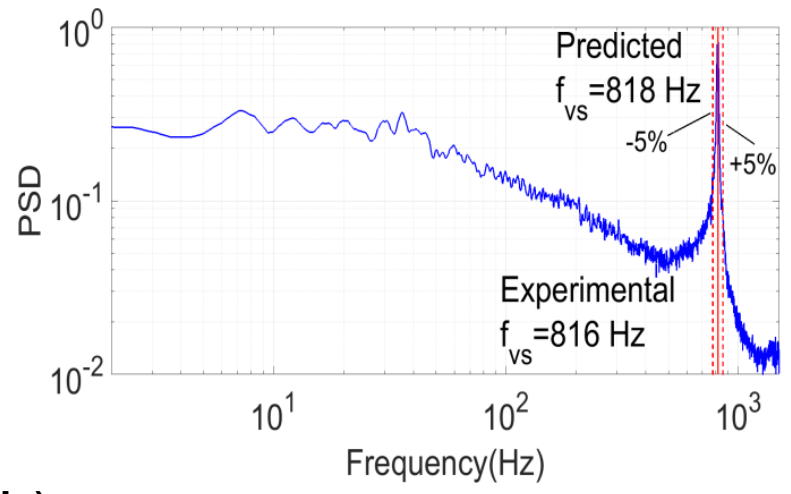

iv)

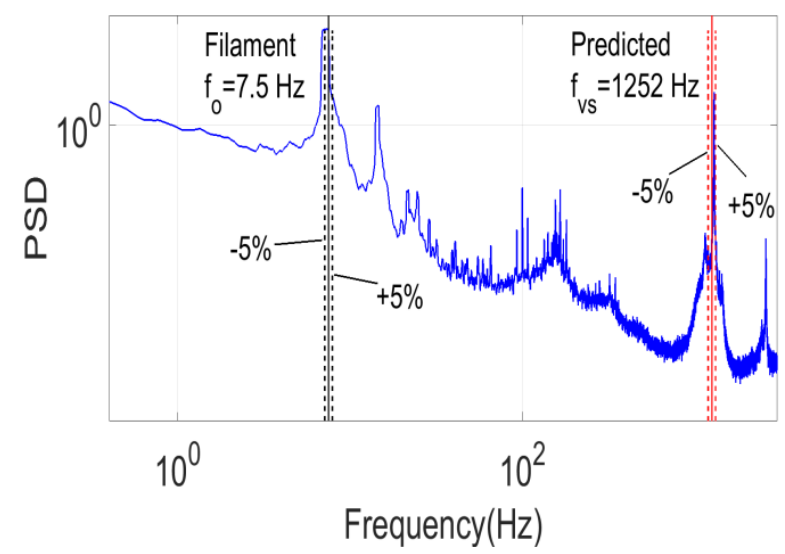

Fig. 16 Measurements in the wake of Filament 11 at $U^{*}=35$. (i) Schematic of filament and locations of measurements. (ii) PSD of support wake. (iii) PSD of filament wake at mid-length. (iv) PSD of filament wake at free-end. 


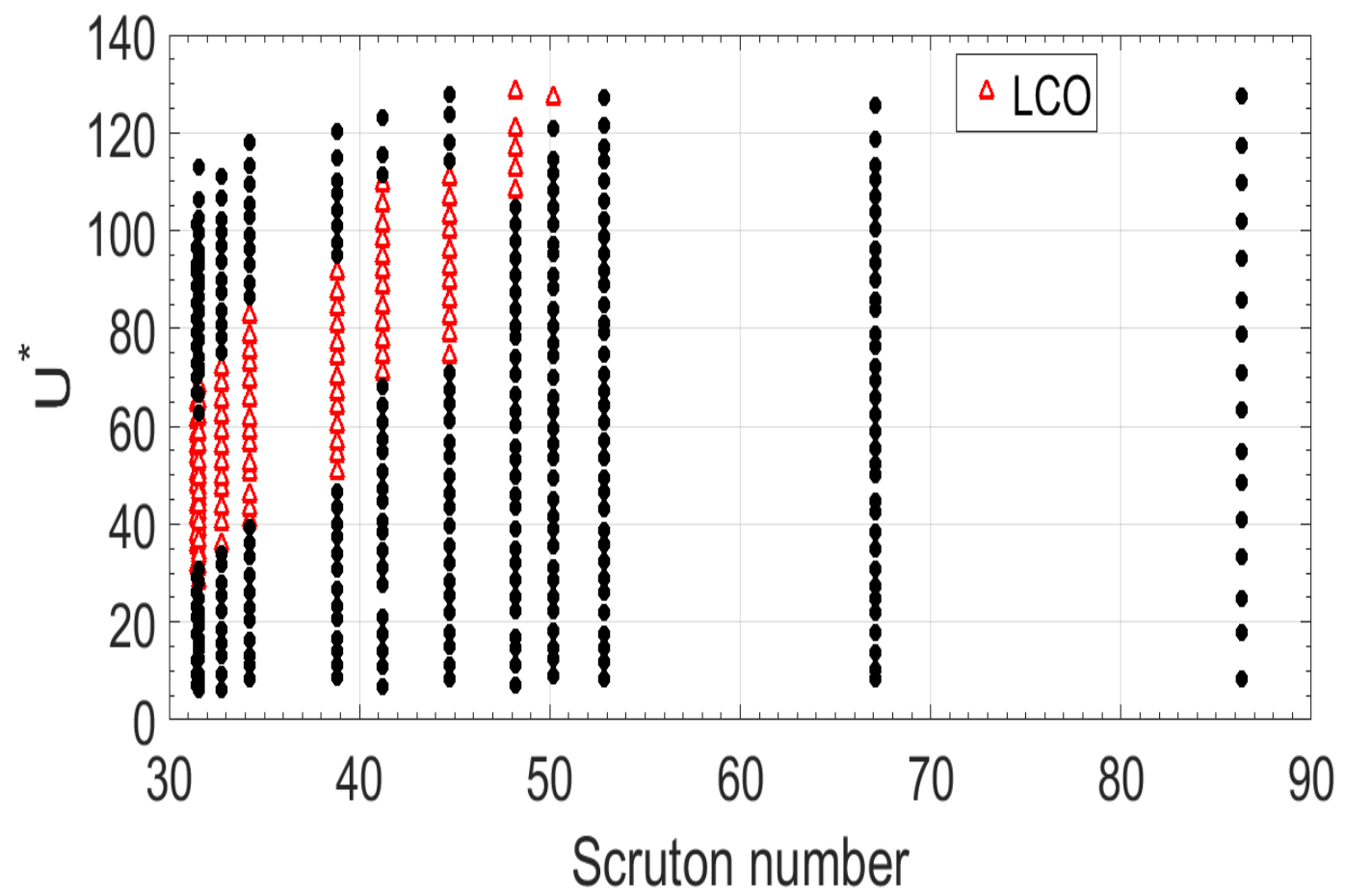

Fig. 17 Stability map: Reduced velocity vs Scruton number. 


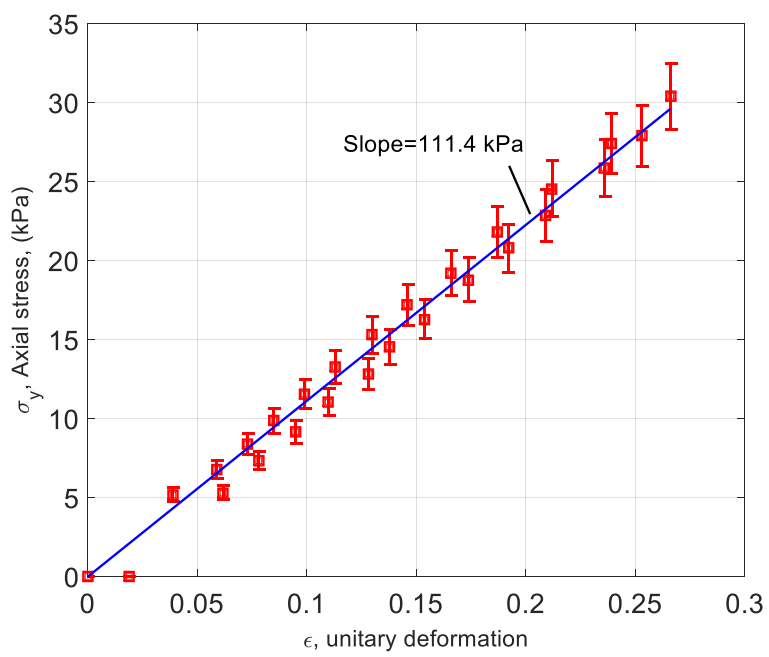

Figure A1. Filament elongation test results: axial stress versus unitary deformation. 


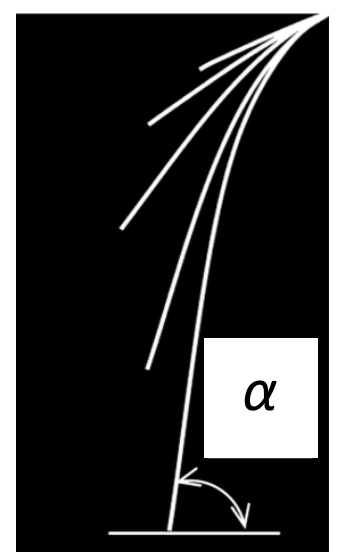

Figure A2 Five equilibrium configurations of a flexible filament under self-weight. 


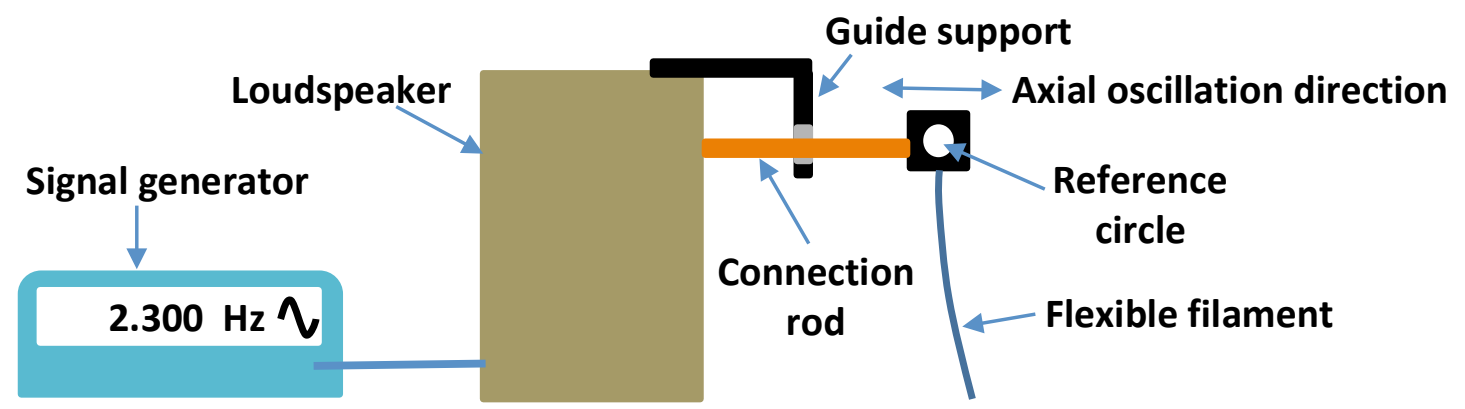

Figure A3 Schematic of the vibration test shaker for free and forced vibration tests on the flexible filaments. 


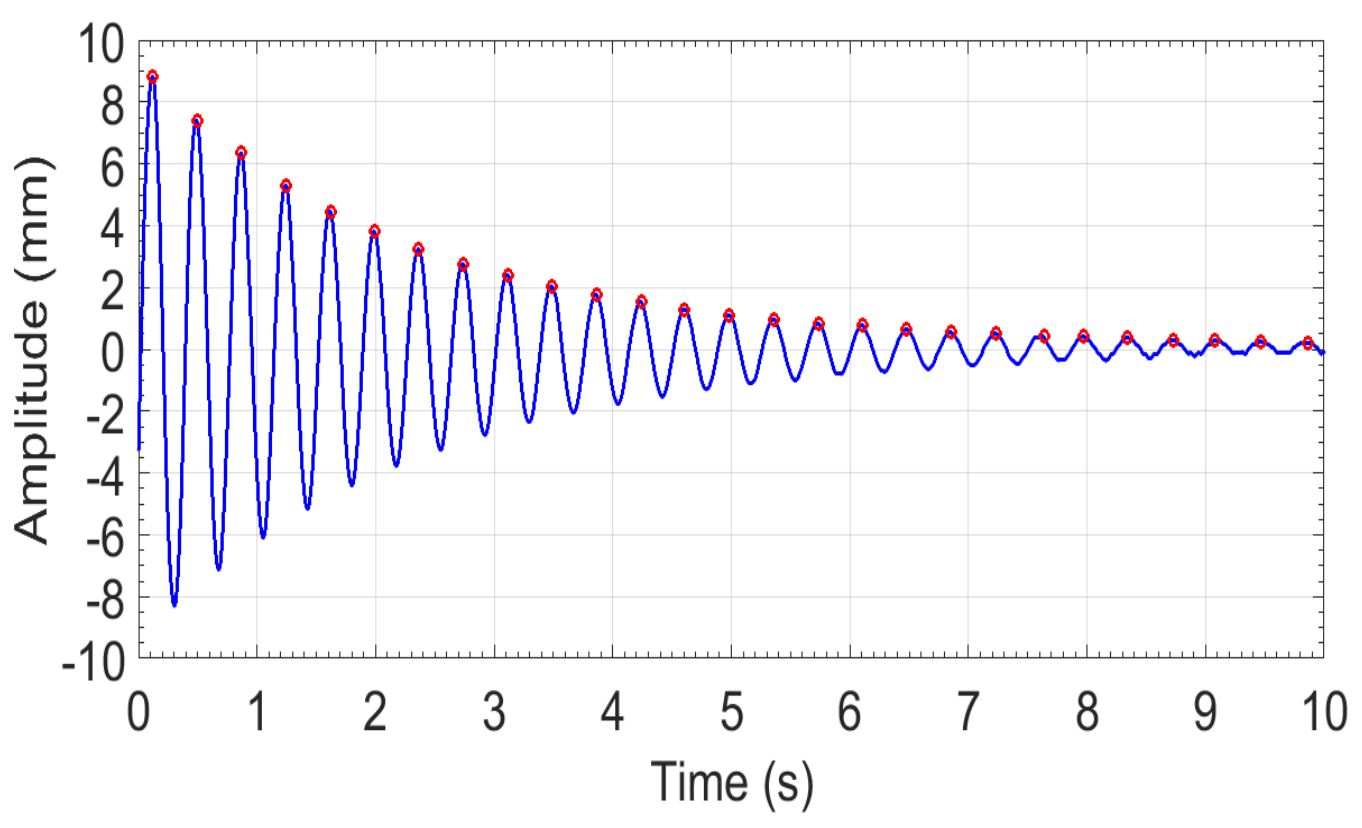

Figure A4 Example of free vibration test results for a filament of $L=55 \mathrm{~mm}$, representative of Filament 1 in Table 1. 

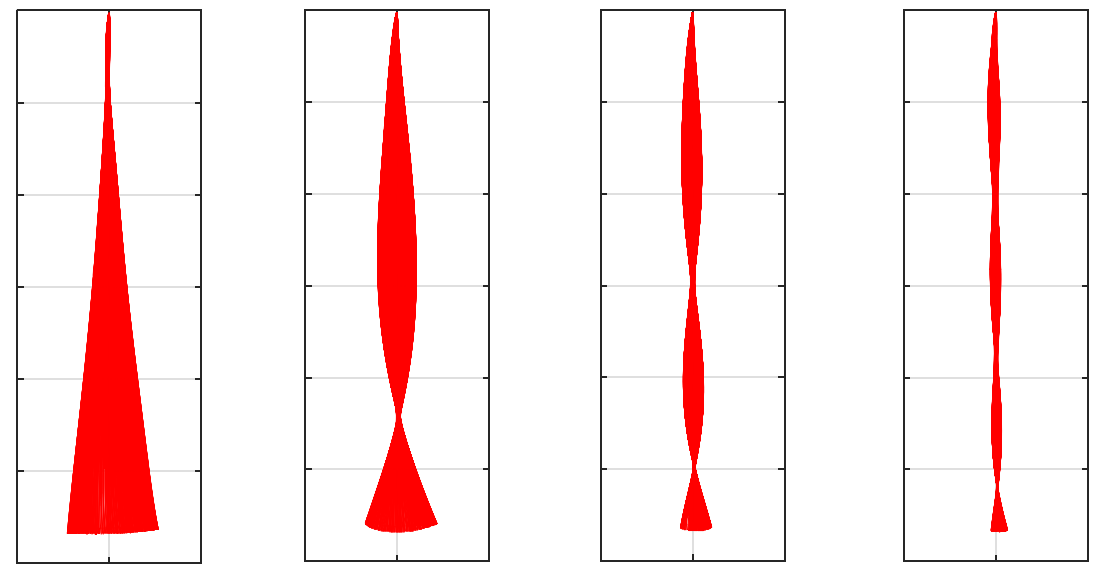

Figure A5 Forced vibration tests: first four vibration modes (from left to right) of the filament of $L=60 \mathrm{~mm}$, representative of Filament 1 in Table 1 . 


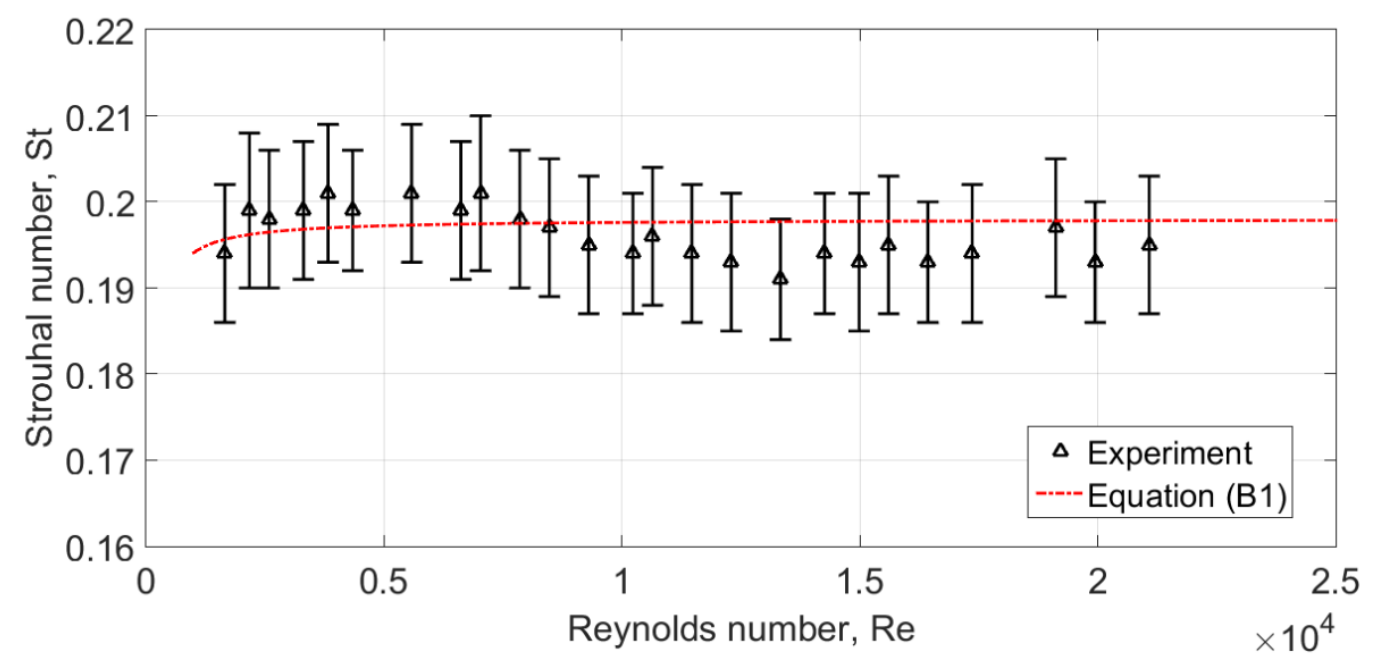

Fig. A6 Test rig validation: Strouhal number vs Reynolds number for a circular cylinder in crossflow. 
Table 1 Geometrical and dynamical properties of the flexible filaments

\begin{tabular}{ccccccc}
\hline $\begin{array}{c}\text { Filament } \\
\text { No. }\end{array}$ & $\begin{array}{c}\boldsymbol{L} \\
(\mathbf{m m})\end{array}$ & $\begin{array}{c}\boldsymbol{f}_{\boldsymbol{n} 1} \\
(\boldsymbol{H z})\end{array}$ & $\begin{array}{c}\boldsymbol{f}_{\boldsymbol{n} 2} \\
(\boldsymbol{H z})\end{array}$ & $\begin{array}{c}\boldsymbol{f}_{\boldsymbol{n} 3} \\
(\boldsymbol{H z})\end{array}$ & $\begin{array}{c}\boldsymbol{f}_{\boldsymbol{n} 4} \\
(\mathbf{H z})\end{array}$ & $\boldsymbol{\zeta}$ \\
\hline $\mathbf{1}$ & 60.0 & 2.6 & 7.0 & 14.5 & 25.2 & 0.0241 \\
$\mathbf{2}$ & 55.0 & 2.7 & 7.5 & 16.4 & 28.1 & 0.0240 \\
$\mathbf{3}$ & 50.0 & 2.8 & 8.1 & 18.2 & 32.5 & 0.0241 \\
$\mathbf{4}$ & 45.0 & 3.1 & 8.7 & 19.5 & 35.0 & 0.0250 \\
$\mathbf{5}$ & 40.0 & 3.3 & 9.9 & 23.0 & 41.0 & 0.0261 \\
$\mathbf{6}$ & 35.0 & 3.7 & 12.0 & 28.2 & 48.0 & 0.0297 \\
$\mathbf{7}$ & 32.0 & 4.0 & 13.2 & 32.0 & - & 0.0315 \\
$\mathbf{8}$ & 29.0 & 4.2 & 14.7 & 36.0 & - & 0.0342 \\
$\mathbf{9}$ & 27.5 & 4.5 & 18.0 & 41.0 & - & 0.0368 \\
$\mathbf{1 0}$ & 26.5 & 4.7 & 19.0 & 44.0 & - & 0.0383 \\
$\mathbf{1 1}$ & 25.0 & 4.9 & 22.0 & 50.0 & - & 0.0404 \\
$\mathbf{1 2}$ & 22.5 & 5.6 & 27.5 & - & - & 0.0513 \\
$\mathbf{1 3}$ & 20.0 & 6.5 & 34.0 & - & - & 0.0660 \\
\hline
\end{tabular}

